\title{
Attention alters uncertainty but not appearance
}

Keith A. Schneider ${ }^{1,2,3, *}$, Anahit Grigorian ${ }^{1}$

${ }^{1}$ Department of Biology, York University, Toronto, ON, Canada

${ }^{2}$ Centre for Vision Research, York University, Toronto, ON, Canada

${ }^{3}$ Department of Psychological \& Brain Sciences, University of Delaware, Newark, DE 19716

*Correspondence to: keithas@udel.edu 


\begin{abstract}
We measured subjects' decision confidence to determine whether paying attention to a stimulus altered its appearance or merely influenced the response decision mechanism. In three separate experiments, subjects judged the relative appearance of two Gabor patches, one of which was cued, that differed in contrast and reported whether they were certain of each response. In Experiments 1 and 2, subjects performed a comparative judgment, reporting which stimulus had the higher contrast, and in Experiment 3, subjects performed an equality judgment, reporting whether the two stimuli had the same or different contrast. Experiments 1 and 3 employed an exogenous attentional pre-cue, and Experiment 2 used a non-attentional post-cue, with explicit instructions to choose the cued target when uncertain. We modeled the subjects' confidence as the totality of the information present in each trial, which included the contrast of each target stimulus, the decision function based on the difference between their contrasts, and the boost in information caused by attention. In Experiments 1 and 2, the cues caused subjects to more likely report that the cued stimulus had higher contrast. In Experiment 1, attention shifted the subjects' confidence distribution, while the post-cue in Experiment 2 did not. In Experiment 3, attention did not affect the contrast judgment but did shift the confidence distribution. We thus report interesting disconnects between confidence and decisions, which were predicted by our model. We conclude that attention acts early in the decision process, but without overwriting the veridical contrast appearance, which is still available to subjects.
\end{abstract}




\section{Introduction}

Does paying attention to an object change the way it looks? This question was investigated in the earliest days of experimental psychology and is still controversial today. One study concluded that an exogenous attentional cue could alter the luminance contrast appearance of a subsequently presented stimulus, causing it to appear higher in contrast when compared to uncued targets (Carrasco et al., 2004). Other studies, employing a variety of different stimuli and experiments, show that, because the effects of attention depend on the type of decision made, and because attention does not alter the ability to make veridical comparisons about appearance, attention does not alter appearance but instead influences the decision mechanisms used when reporting appearance (Itthipuripat et al., 2019; Kerzel et al., 2010; Linares et al., 2019; Schneider, 2006; Schneider \& Komlos, 2008; Schneider \& Malik, 2021; Zhou et al., 2018). We have proposed that attention increases the salience of a stimulus without altering its appearance (Beck \& Schneider, 2017). Increasing salience has of a target, for example making it pop out in an array of competing stimuli, has been shown to have an equivalent effect on decisions as an actual increase in contrast (Kerzel et al., 2011), and also similar to the effects of attention. However, the exact mechanism by which attention operates upon the decision mechanism remains unclear.

Recently, we proposed an uncertainty stealing hypothesis that predicts that, when subjects are uncertain about their perceptual judgment, they would tend to disproportionately allocate their responses to the cued stimulus (Schneider \& Malik, 2021). Thus, if two stimuli appeared equal in contrast but one was cued, and subjects were required to choose which of two apparently equal stimuli had the higher contrast, 
subjects would be maximally uncertain and might tend to disproportionately choose the

more salient cued stimulus. In the present study, we measure uncertainty explicitly to test the uncertainty stealing hypothesis. Contrary to the predictions of the uncertainty stealing hypothesis, we found that attention did not merely promote the disproportional allocation of uncertainty, but rather it shifted the confidence distribution in favor of the cued target, and thus must operate earlier in the decision process, before subjects' form an awareness of their decisions. Even so, subjects were still able to make veridical comparisons of stimulus contrast, and we have observed interesting disconnects between the results of a decision and subjects' confidence about it.

\section{Methods}

Subjects. Fourteen undergraduate subjects were paid to participate in the study. Three subjects were excluded from the analysis: one gave the same response to every trial (S5), one responded randomly in one experiment (S10), and one very rarely indicated uncertainty (S14). We show the data from all subjects, including these 3 excluded subjects, in the Appendix.

All subjects had normal or corrected-to-normal visual acuity, gave their written, informed consent under a protocol approved by the York University Human Participants Review Committee, and were naïve to the purpose of the experiment.

Apparatus. The stimuli were generated on a Macintosh Pro computer (Apple, Inc., Cupertino, CA) using the Matlab (The Math Works, Inc., Natick, MA) programming language and displayed using Psychophysics Toolbox 3 functions (Brainard, 1997; 
Kleiner et al., 2007; Pelli, 1997) on a ViewSonic P225fb monitor (ViewSonic Corp., Walnut, CA) with a refresh rate of $119.6 \mathrm{~Hz}$. The stimuli durations reported below were constrained by the refresh rate and were rounded up to an integral multiple of the $8.4 \mathrm{~ms}$ frame rate. The output channels of the video card were combined with a video attenuator device (Video Switcher, Xiangrui Li, Los Angeles, CA) to enable 12-bit precision in the gray-scale luminance values ( $\mathrm{Li}$ et al., 2003). The gamma function and luminance of the monitor were measured using an LS-100 photometer (Konica Minolta Photo Imaging USA, Mahwah, NJ).

Experiments. Each subject participated in each of three experiments conducted on separate days during the period of November 2012 through January 2013.

Experiment 1: Comparative and uncertainty judgments with attentional pre-cue. The objective of this experiment was to determine whether an exogenous cue shifted subjects' uncertainty in favor of the cued target.

Experiment 2: Comparative and uncertainty judgments with post-cue and explicit bias instructions to choose the cued target when uncertain. This experiment served as a control to measure whether a non-attentional response bias would shift uncertainty in the same manner as an attentional cue (Gallagher et al., 2019).

Experiment 3: Equality and uncertainty judgments with attentional pre-cue. This experiment determined whether subjects retained veridical information about the stimuli contrasts, and whether attention shifted uncertainty in a similar manner in different types of decisions.

These experiments are described further below. 
Visual stimuli. A diagram of the stimulus sequence is shown in Figure 1. Two Gabor stimuli targets, 4 cpd sine gratings, oriented vertically, zero phase and Gaussian envelopes with a standard deviation of $1^{\circ}$, were simultaneously presented for $40 \mathrm{~ms}$ on a uniform gray field. The centers of the targets were located $4^{\circ}$ eccentricity to the right or left of the fixation point. In Experiments 1 and 3,120 ms before the onset of the targets, a $0.3^{\circ}$ diameter black dot was presented for $67 \mathrm{~ms}$ at the location $1.5^{\circ}$ directly above the center of one of the two targets. In Experiment 2, the onset of this cue stimulus was 500 ms after the target onset (460 ms after the targets disappeared), but the other parameters were the same. The luminance contrasts of the two targets were distinct and were defined as $\frac{L_{1}-L_{2}}{L_{1}+L_{2}}$, where $L_{1}$ is the maximum luminance of the sine wave component of the Gabor and $L_{2}$ is the minimum. The mean luminance of the sine wave components equaled the background luminance, $85 \mathrm{~cd} / \mathrm{m}^{2}$. The contrast of the cued target was $25 \%$, and the contrast of the uncued target was chosen from 61 different evenly distributed logarithmic range of contrasts spanning \pm 1.5 natural log units relative to the cued target contrast. Three of these contrast levels exceeded the ceiling of $100 \%$ contrast, so the data for those stimuli were removed from the analysis, resulting in 58 uncued target contrasts ranging from 5.6-96.4\%.

Procedure. Subjects were seated alone in a dark room and viewed the display unrestrained from $50 \mathrm{~cm}$. The cue stimulus appeared randomly on the left or right for each trial. Each of the 61 contrast levels of the uncued target were repeated 25 times, for a total of 1525 trials, randomly distributed throughout the session. 
The task of the subjects on each trial was to determine, In Experiments 1 and 2 (comparative judgment), which target had the higher contrast, and in Experiments 3 (equality judgment), whether the two targets were equal in contrast. In Experiment 2, a decision bias was explicitly introduced by instructing the subjects to always choose the post-cued stimulus if they were uncertain which of the two stimuli had the higher contrast. For the comparative judgment, subjects pressed the left arrow key on the keyboard to indicate that the target on the left had the higher contrast or the right arrow key to indicate that the target on the right had higher contrast. For the equality judgment, subjects pressed the S key to indicate that the two targets had equal ("same") contrast or the D key to indicate that the two targets had unequal ("different") contrast.

In all three experiments, in addition to the relative contrast judgment, subjects were also required to indicate the certainty of each of their responses. While still holding down the first response key, subjects pressed the up-arrow key if they were certain about their response or the down-arrow key if they were uncertain. Subjects had unlimited time to respond. The cue stimulus for the next trial appeared $0.5-1 \mathrm{~s}$ after the response was made.

Each experimental sessions lasted approximately one hour, during which the observers were automatically allowed to rest and break fixation after every 50 trials, resuming fixation and the experiment when ready. Each subject began each experiment with a practice run with the experimenter in the room, demonstrating understanding of the instructions of that experiment and the ability to perform the task. 
Decision model. The equivalent contrasts of the cued targets were determined by fitting previously derived psychometric functions (García-Pérez \& Alcalá-Quintana, 2019; Schneider, 2006; Schneider \& Bavelier, 2003; Schneider \& Komlos, 2008) to the subjects' responses. A cumulative normal distribution, $\Psi=1-\Phi\left(\frac{\Delta c+\alpha}{\sigma}\right)$ was fit to the comparative judgment responses, and a difference of cumulative normal distributions, $\Psi=\Phi\left(\frac{\tau-\Delta c-\alpha}{\sigma}\right)-\Phi\left(\frac{-\tau-\Delta c-\alpha}{\sigma}\right)$ to the equality judgment responses (also see DeCarlo, 2013), where $\Phi(x) \equiv \frac{1}{2 \pi} \int_{-\infty}^{x} e^{-u^{2} / 2} d u$ is the cumulative normal distribution, $\tau$ is the contrast difference criterion (which controls the amplitude of the function), $\Delta c$ is actual difference in contrast between the cued target and the uncued target, $\sigma^{2}$ is the variance of the contrast difference and $\alpha$ is the potential boost in perceived contrast of the cued target relative to the uncued target. $\alpha$ is the difference in contrast where the point of subjective equality (PSE) occurs: the point at which the psychometric function for the comparative judgment crosses $50 \%$, or the maximal point of the equality judgment function. Each parameter has units of logarithmic contrast. The benefit of the equality judgment is that a subject's decision criterion (amplitude) is orthogonal to an attentional change in contrast (central tendency), so the two effects can be distinguished; in the comparative judgment these parameters are degenerate (Schneider \& Bavelier, 2003; Schneider \& Komlos, 2008).

Uncertainty model. We sought to develop a meaningful model of confidence for the two stimulus decisions. We operationalized confidence $(C)$ as being proportional to the total amount of information present during each experimental trial, which was the sum of three 
components: 1. intrinsic information from the stimuli themselves, 2 . information in the decision variable, which is inversely proportional to the rate of change of the psychometric function, and 3. any increase in information caused by the attentional cue. The point(s) of maximum uncertainty $(U \equiv 1-C)$ should correspond to the maximum slope, or rate of change, of the psychometric function. The derivative of the psychometric functions are $\frac{d \Psi}{d \Delta c}=-\frac{1}{\sigma \sqrt{2 \pi}} e^{-\frac{1}{2}\left(\frac{\Delta c+\alpha}{\sigma}\right)^{2}}$ for the comparative judgment and the bimodal $\frac{d \Psi}{d \Delta c}=-\frac{1}{\sigma \sqrt{2 \pi}}\left(e^{-\frac{1}{2}\left(\frac{-\tau-\Delta c-\alpha}{\sigma}\right)^{2}}-e^{-\frac{1}{2}\left(\frac{-\tau-\Delta c-\alpha}{\sigma}\right)^{2}}\right)$ for the equality judgment, where the parameters are the same as above, except $\alpha$ here represents the increase in information due to the attentional cue, and $\tau$ is the subjective threshold criterion that reflects what each subject considers "certain" or "uncertain". Although explicitly using the derivative is a novel derivation of the functional form of the uncertainty distribution, previous studies have done so de facto, although they did not provide any explanation for doing so. other than that the functions fit the shape of the data (Caziot \& Mamassian, 2021; Gallagher et al., 2019, 2021; Maldonado Moscoso et al., 2020). For uncertainty responses in the equality judgment that did not exhibit the bimodal distribution predicted by its derivative, the centroid of the response distribution was estimated by fitting the comparative judgment uncertainty function instead. We speculate that subjects might become more uncertain as the visibility of the lower contrast stimulus decreased, similar to the non-zero asymptote at low contrast observed in a previous study (Schneider, 2011). Therefore, we assumed that the information intrinsic to the stimuli would be proportional to $c$, where $c$ is the logarithm of stimulus contrast. Since only the uncued stimuli varied in contrast, we added a linear term to represent the stimulus information. Therefore, the 
complete uncertainty function was $U=-\frac{d \Psi}{d \Delta c}+x_{0}+x_{1} \Delta \mathrm{c}$, where $x_{0}$ and $x_{1}$ are the constant and linear slope parameters.

\section{Results}

A typical pattern of responses is shown in Figure 2 for a comparative judgment (upper panels) and equality judgment (lower panels) and their associated confidence reports (right panels). As shown in Figure 3 (blue bars), for the comparative judgments, both the attentional pre-cue (Experiment 1) and the post-cue and bias instructions (Experiment 2) served to shift the PSE (the $\alpha$ parameter) of the psychometric functions, such that an uncued stimulus needed to be $38.5 \%$ contrast $(\alpha=0.433 \pm 0.087$, mean $\pm \mathrm{SEM}, p=$ .00077 , two-tailed $t$-test $)$ or $35.0 \%$ contrast $(\alpha=0.338 \pm 0.045, p=.000029)$, respectively, to be judged equivalent to the cued $25 \%$ contrast stimulus $\left(c^{\prime}=c e^{\alpha}\right)$. As with previous results in the literature, the attentional pre-cue did not change the PSE significantly from 0 for the equality judgment in Experiment $3(\alpha=0.092 \pm 0.080, p=$ .30), with subjects judging the veridical contrast for the cued stimulus. However, within subjects, the PSEs determined from the comparative and equality judgments with the attentional pre-cue (Experiments 1 and 3) were significantly correlated (Figure 4a, $r=$ $.63, p=.035$; also significantly different from each other, paired $t$-test, $t_{10}=4.57, p=$ $.001)$, whereas the post-cue bias PSEs (Experiment 2) were not correlated with either $(r=$ $.21, p=.54$ and $r=.16, p=.64$ for Exps. 1 and 3, respectively).

In some subjects' confidence reports, there was a linear trend such that they were more uncertain for the lower contrast uncued targets. However, across subjects and experiments, this linear term was only significantly different from zero in Experiment 3 , 
slope $=0.271 \pm 0.084, p=.012$. However, in Experiment 2, there was a significant correlation across subjects between the slope and the PSE of the contrast judgment (Figure $4 \mathrm{~b}, r=.79, p=.0038$ ) and between the slope and the centroid of the uncertainty distribution $(r=.67, p=.022)$. This was expected because the subjects were instructed in this experiment to indicate that the cued target had the higher contrast when they were uncertain about their judgment. Thus, the more uncertain a subject was, the higher would be the PSE contrast. The linear term was not correlated with the centroid of the uncertainty distribution across subjects in Experiments 1 or $3(r=-.31, p=.36$ and $r=$ $-.10, p=.77$, respectively), suggesting that the subjects' increase in uncertainty when comparing low-contrast uncued targets had limited bearing on the results.

Accounting for the linear trend, the point of maximum uncertainty was found to differ based on the type of cue used in each experiment (Fig. 3, red bars). The attentional pre-cues in Experiment 1 and 3 had similar effects, significantly moving the point of maximum uncertainty away from the low-contrast cues $(\alpha=0.549 \pm 0.075, p=.000039$ and $\alpha=0.288 \pm 0.071, p=.0031$, respectively, though significantly different from each other within subject, $t_{10}=2.77, p=.020$, paired $t$-test). The post-stimulus cue and bias instructions employed in Experiment 2, however, caused a different pattern of results. While the cue and instructions moved the PSE towards higher contrasts, similar to the attentional pre-cue in Experiment 1, the effects on the point of maximum uncertainty were quite different — the subjects' uncertainty judgments were not moved in the direction of higher contrast but were instead largest when the uncued target was of a similar contrast to the cued target (see Fig. $3, \alpha$ smaller but significantly less than $0, \alpha=$ $-0.081 \pm 0.031, p=.031)$. Interestingly, in all 3 experiments, the PSE in the contrast 
judgment was significantly correlated with the centroid or point of maximum uncertainty in the confidence report (Fig. 4, $r=.63, p=.035 ; r=.80, p=.0034$; and $r=.67, p=.023$ for Experiments 1-3, respectively).

\section{Discussion}

The results show that in comparisons between two stimuli, exogenous attention to one of them can shift subjects' confidence ratings in favor of the cued stimulus. For both the comparative and equality judgments in Experiments 1 and 3, the subjects' points of maximum uncertainty were shifted towards the higher contrast stimuli. That is, the subjects were not maximally uncertain when the cued target was the same contrast as the uncued target, but rather when the uncued target had higher contrast. Landry, et al. (2021) concluded that attention does not directly affect subjective perception but rather indirectly affects confidence by increasing perceptual evidence and lowering decision criteria. As we hypothesized previously (Beck \& Schneider, 2017), this increased perceptual evidence can manifest as increased salience and therefore make a target more competitive against other stimuli in the decision process.

This study was designed measure uncertainty directly to test our previously proposed uncertainty stealing hypothesis (Schneider \& Malik, 2021), in which subjects would tend to disproportionally allocate uncertain decisions in favor of the higher salience attended target (Beck \& Schneider, 2017; Schneider \& Malik, 2021). In the comparative judgment, subjects are forced to choose which target has the higher contrast even when the two targets are apparently equally, when the subjects should be maximally uncertain. However, this is not what we found. Attention shifted the confidence 
distribution to favor the attended stimulus, i.e. subjects were maximally uncertain when the cued target was lower in contrast than the uncued target. Interestingly, in the equality judgment, attention similarly shifted the confidence distribution, but subjects were nonetheless still able to perform the veridical contrast comparison. We also found that in some subjects, uncertainty increased when the uncued target had lower contrast, compared to when it had higher contrast but was equally distinct from the cued target.

Taken together, the confidence ratings support our proposed model that confidence depends on the totality of the evidence available in that trial. Subjects were more confident when the targets were further apart in contrast and also when either target was more salient, either by the intrinsic contrast (higher contrast gives more information) or through the action of attention upon the cued target (attention increases information, e.g. Carrasco et al., 2002). This simple model fit the subjects' uncertainty distributions well and explained the main features of the data: 1 . subjects' uncertainty increases as the decision becomes more difficult; 2 . subject's uncertainty tends to increase as the stimuli decrease in contrast (less information about each stimulus); and 3. attention decreases uncertainty, as attention provides more information about the cued target. For the last point, previous studies have suggested that exogenous attention has an additive effect in terms of effective contrast (Cutrone et al., 2014; Schneider, 2006).

We modeled the information available from the difference between the two targets as the derivative of the psychometric function, because the available information is smallest when the rate of change of the psychometric function is the largest. We were able to test this model in Experiment 3, where the derivative of the equality judgment was a bimodal function. This was observed in some but not all subjects - although these 
subjects did exhibit the same shift as the others in the centroid of their uncertainty distribution - so the test of the model had mixed results. We do not know whether some subjects had a different method of subjectively determining certainty in this experiment, or whether the bimodality was simply lost in the noise.

We observed several disconnects between confidence and the decision. First, in all three experiments, the uncertainty distribution was asymmetric, with higher uncertainty when the uncued target had lower contrast. This was true even though the psychometric functions themselves were symmetric and did not exhibit any differences between low and high contrast uncued targets. This was predicted by our model, because information available about the stimuli themselves, which decreases with decreasing contrast, should influence the reported uncertainty. Second, in Experiment 2, the postcue shifted the decision but did not affect certainty; conversely, in Experiment 3, the exogenous cue shifted uncertainty but did not shift the decision. In Experiment 1, the exogenous cue similarly shifted both the decision and uncertainty. Because the confidence judgments are the subjects' subjective assessments of their decisions, it is not unexpected that they do not accurately reflect the decision. The most famous example of this is blindsight, in which subjects report being completely uncertain but are nonetheless able to make accurate decisions (Weiskrantz, 1996).

Several previous studies have investigated the effects of attentional cues on single targets in discrimination or detection tasks. For endogenous attentional cues, there is disagreement whether the cues increased (Kurtz et al., 2017), decreased (Recht et al., 2021) or had no effect on confidence (Landry et al., 2021; Wilimzig et al., 2008). These studies all agreed, however, that the exogenous cues, such as those used in the present 
study, had no effect on confidence ratings for single stimuli. The present study differs from these studies in that we were measuring the effects of attentional cues on the comparison between two stimuli, one of which was cued and the other not. We measured uncertainty directly, and we did observe that the exogenous cues shifted the uncertainty in favor of the cued target. However, attention acted earlier in the decision process than we expected, not merely causing uncertainty to be disproportionally allocated to the cued target, but rather, shifting the entire confidence distribution.

Based on the results of the present study, we were able to narrow down the point at which attention affected the stimulus-response process. We know that attention did not alter the encoding of stimulus contrast, because subjects were still able to make the veridical contrast comparisons in Experiment 3. Even though both attention and contrast can increase neural firing rates, attention does not overwrite contrast - attention and contrast remain separable codes in the primary visual cortex (Pooresmaeili et al., 2010). However, we did observe that attention acted early in the decision process. Even though the attentional cue in Experiment 1 and the post-stimulus bias in Experiment 2 produced indistinguishable psychometric functions, the confidence ratings differentiated the two experiments; attention shifted the uncertainty ratings in Experiment 1, whereas the poststimulus response bias in Experiment 2 did not. We are therefore able to conclude that attention manipulated the decision mechanism while the decisions were being made, in doing so, altering the subjects' assessments of their decisions. The response bias in Experiment 2 did not alter the certainty and we can conclude that it acted after the perceptual decision. The fact that the two types of cues produced indistinguishable 
psychometric functions underscores the fact that shifts in the PSE of a comparative judgment are not sufficient to conclude that attention alters appearance.

\section{Conclusion}

Taken together, the three experiments demonstrated that exogenous attention to one stimulus manipulated uncertainty in a comparison between two stimuli. However, there was a disconnect between subjects' confidence reports and their decisions. Confidence was based on the totality of the evidence available in a decision, which was increased by attention. Measuring confidence determined the locus of attentional action to occur early in the decision process. Attention increased the salience of a stimulus and increased subjects' confidence in their decision, even while not altering appearance. 


\section{Figure captions}

Figure 1. Stimulus sequence. Subjects fixated for 500-1000 ms, after which a cue appeared at $4^{\circ}$ eccentricity on the left or right. In Experiments 1 and 3, the cue disappeared after $67 \mathrm{~ms}$, and $120 \mathrm{~ms}$ after the cue onset, two Gabor grating stimuli appeared centered at the same eccentricity, one of which was slightly below the location at which the cue had appeared. After $40 \mathrm{~ms}$, the stimuli disappeared, and subjects reported their relative contrasts. In Experiment 2, the cue appeared $500 \mathrm{~ms}$ after the onset of the Gabor gratings, $460 \mathrm{~ms}$ after they disappeared.

Figure 2. Typical responses from one subject. The abscissa represents the natural logarithm of the ratio of the cued target contrast to the uncued target contrast. The subjects performed two different types of judgments (left panels). In the comparative judgment (upper panels), the subjects reported which of the two targets appeared to have the higher contrast, and the ordinate represents the fraction of responses in which a subject indicated the cued target had higher contrast. The circular markers indicate the mean response for 25 repetitions. From the model fit to the distribution of responses, indicated by the solid gray line, the point of subjective equality (PSE) was determined as the point at which the subject was equally likely to report either target as having the higher contrast $(P=1 / 2)$. In the equality judgment (lower panels), the subjects reported whether the two stimuli were equal in contrast; the ordinate represents the fraction of affirmative responses. The PSE was determined from the model fit as the point of maximal equality. For all judgments, subjects also rated their confidence, responding whether they were certain or uncertain of their response on each trial (right panels). The 
confidence reports were fit to a model that was the derivative of the comparative or equality judgment.

Figure 3. For all 3 experiments, the mean results for across subject are shown for the $\alpha$ parameter, which is the PSE for the decisions (blue bars) and centroid/maximum of the uncertainty distribution (red bars). The error bars indicate the $95 \%$ confidence interval of the mean. $* p<.05, * * p<.01, * * * p<.001$

Figure 4. Correlations. a. The PSEs $(\alpha)$ for the attentional pre-cue experiments (Experiment 1 and 3) were found to be significantly correlated with each other across subjects. b. For Experiment 2 (post-cue bias instructions), the PSE ( $\alpha$ ) was found to be significantly correlated with the linear trend in the reported uncertainty.

Figure 5. Correlations between the decision PSEs and uncertainty distributions. For all 3 experiments, the measured PSE for each contrast judgment ( $\operatorname{PSE} \alpha$ ) was found to be significantly correlated with the centroid/maximum of the uncertainty distribution (uncertainty $\alpha$ ). 


\section{References}

Beck, J., \& Schneider, K. A. (2017). Attention and Mental Primer. Mind \& Language, 32(4), 463-494. https://doi.org/10.1111/mila.12148

Brainard, D. H. (1997). The Psychophysics Toolbox. Spatial Vision, 10(4), 433-436. https://doi.org/10.1163/156856897X00357

Carrasco, M., Ling, S., \& Read, S. (2004). Attention alters appearance. Nature Neuroscience, 7(3), 308-13. https://doi.org/10.1167/12.9.1387

Carrasco, M., Williams, P. E., \& Yeshurun, Y. (2002). Covert attention increases spatial resolution with or without masks: support for signal enhancement. Journal of Vision, 2(6), 467-479. https://doi.org/10.1167/2.6.4

Caziot, B., \& Mamassian, P. (2021). Perceptual confidence judgments reflect selfconsistency. Journal of Vision, 21(12), 8-8. https://doi.org/10.1167/JOV.21.12.8

Cutrone, E. K., Heeger, D. J., \& Carrasco, M. (2014). Attention enhances contrast appearance via increased input baseline of neural responses. Journal of Vision, 14(14), 16-16. https://doi.org/10.1167/14.14.16

DeCarlo, L. T. (2013). Signal detection models for the same-different task. Journal of Mathematical Psychology, 57(1-2), 43-51. https://doi.org/10.1016/j.jmp.2013.02.002

Gallagher, R. M., Suddendorf, T., \& Arnold, D. H. (2019). Confidence as a diagnostic tool for perceptual aftereffects. Scientific Reports 2019 9:1, 9(1), 1-12. https://doi.org/10.1038/s41598-019-43170-1

Gallagher, R. M., Suddendorf, T., \& Arnold, D. H. (2021). The implied motion aftereffect changes decisions, but not confidence. Attention, Perception, and 
Psychophysics, 83(8), 3047-3055. https://doi.org/10.3758/s13414-021-02331-z

García-Pérez, M. Á., \& Alcalá-Quintana, R. (2019). The Do's and Don’ts of

Psychophysical Methods for Interpretability of Psychometric Functions and Their

Descriptors. Spanish Journal of Psychology, 2019, 1-30.

https://doi.org/10.1017/sjp.2019.49

Itthipuripat, S., Chang, K.-Y., Bong, A., \& Serences, J. T. (2019). Stimulus visibility and uncertainty mediate the influence of attention on response bias and visual contrast appearance. Journal of Vision, 19(14), 8. https://doi.org/10.1167/19.14.8

Kerzel, D., Schönhammer, J., Burra, N., Born, S., \& Souto, D. (2011). Saliency changes appearance. PLoS ONE, 6(12), e28292.

https://doi.org/10.1371/journal.pone.0028292

Kerzel, D., Zarian, L., Gauch, A., \& Buetti, S. (2010). Large effects of peripheral cues on appearance correlate with low precision. Journal of Vision, 10(11), 26. https://doi.org/10.1167/10.11.26

Kleiner, M., Brainard, D., Pelli, D., Ingling, A., Murray, R., \& Broussard, C. (2007). Perception. In Perception (Vol. 36, Issue 14). [Pion Ltd.]. https://nyuscholars.nyu.edu/en/publications/whats-new-in-psychtoolbox-3

Kurtz, P., Shapcott, K. A., Kaiser, J., Schmiedt, J. T., \& Schmid, M. C. (2017). The Influence of Endogenous and Exogenous Spatial Attention on Decision Confidence. Scientific Reports, 7(1). https://doi.org/10.1038/S41598-017-06715-W

Landry, M., Da Silva Castanheira, J., Sackur, J., \& Raz, A. (2021). Investigating how the modularity of visuospatial attention shapes conscious perception using type I and type II signal detection theory. Journal of Experimental Psychology. Human 
Perception and Performance, 47(3), 402-422. https://doi.org/10.1037/XHP0000810

Li, X., Lu, Z.-L., Xu, P., Jin, J., \& Zhou, Y. (2003). Generating high gray-level resolution monochrome displays with conventional computer graphics cards and color monitors. Journal of Neuroscience Methods, 130(1), 9-18.

https://doi.org/10.1016/S0165-0270(03)00174-2

Linares, D., Aguilar-Lleyda, D., \& López-Moliner, J. (2019). Decoupling sensory from decisional choice biases in perceptual decision making. ELife, 8, 1-16. https://doi.org/10.7554/eLife.43994

Maldonado Moscoso, P. A., Cicchini, G. M., Arrighi, R., \& Burr, D. C. (2020). Adaptation to hand-tapping affects sensory processing of numerosity directly: evidence from reaction times and confidence. Proceedings of the Royal Society B, 287(1927). https://doi.org/10.1098/RSPB.2020.0801

Pelli, D. G. (1997). The VideoToolbox software for visual psychophysics: transforming numbers into movies. Spatial Vision, 10(4), 437-442. https://doi.org/10.1163/156856897X00366

Pooresmaeili, A., Poort, J., Thiele, A., \& Roelfsema, P. R. (2010). Separable codes for attention and luminance contrast in the primary visual cortex. Journal of Neuroscience, 30(38), 12701-11. https://doi.org/30/38/12701 [pii] 10.1523/JNEUROSCI.1388-10.2010

Recht, S., de Gardelle, V., \& Mamassian, P. (2021). Metacognitive blindness in temporal selection during the deployment of spatial attention. Cognition, 216. https://doi.org/10.1016/J.COGNITION.2021.104864

Schneider, K. A. (2006). Does attention alter appearance? Perception \& Psychophysics, 
68(5), 800-814. https://doi.org/10.3758/BF03193703

Schneider, K. A. (2011). Attention alters decision criteria but not appearance: a reanalysis of Anton-Erxleben, Abrams, and Carrasco (2010). Journal of Vision, 11(13), 7. https://doi.org/10.1167/11.13.7

Schneider, K. A., \& Bavelier, D. (2003). Components of visual prior entry. Cognitive Psychology, 47(4), 333-366. https://doi.org/10.1016/S0010-0285(03)00035-5

Schneider, K. A., \& Komlos, M. (2008). Attention biases decisions but does not alter appearance. Journal of Vision, 8(15), 3, 1-10. https://doi.org/10.1167/8.6.1094

Schneider, K. A., \& Malik, I. (2021). A three-response task reveals how attention alters decision criteria but not appearance. Journal of Vision, 21(1), 1-16. https://doi.org/10.1167/jov.21.5.30

Weiskrantz, L. (1996). Blindsight revisited. Current Opinion in Neurobiology, 6(2), 215220. https://doi.org/10.1016/S0959-4388(96)80075-4

Wilimzig, C., Tsuchiya, N., Fahle, M., Einhäuser, W., \& Koch, C. (2008). Spatial attention increases performance but not subjective confidence in a discrimination task. Journal of Vision, 8(5), 7-7. https://doi.org/10.1167/8.5.7

Zhou, L. F., Buetti, S., Lu, S., \& Cai, Y. C. (2018). Attentional effect on contrast appearance: From enhancement to attenuation. Journal of Experimental Psychology: Human Perception and Performance, 44(5), 806-817. https://doi.org/10.1037/xhp0000499 


\section{Figures}

Figure 1

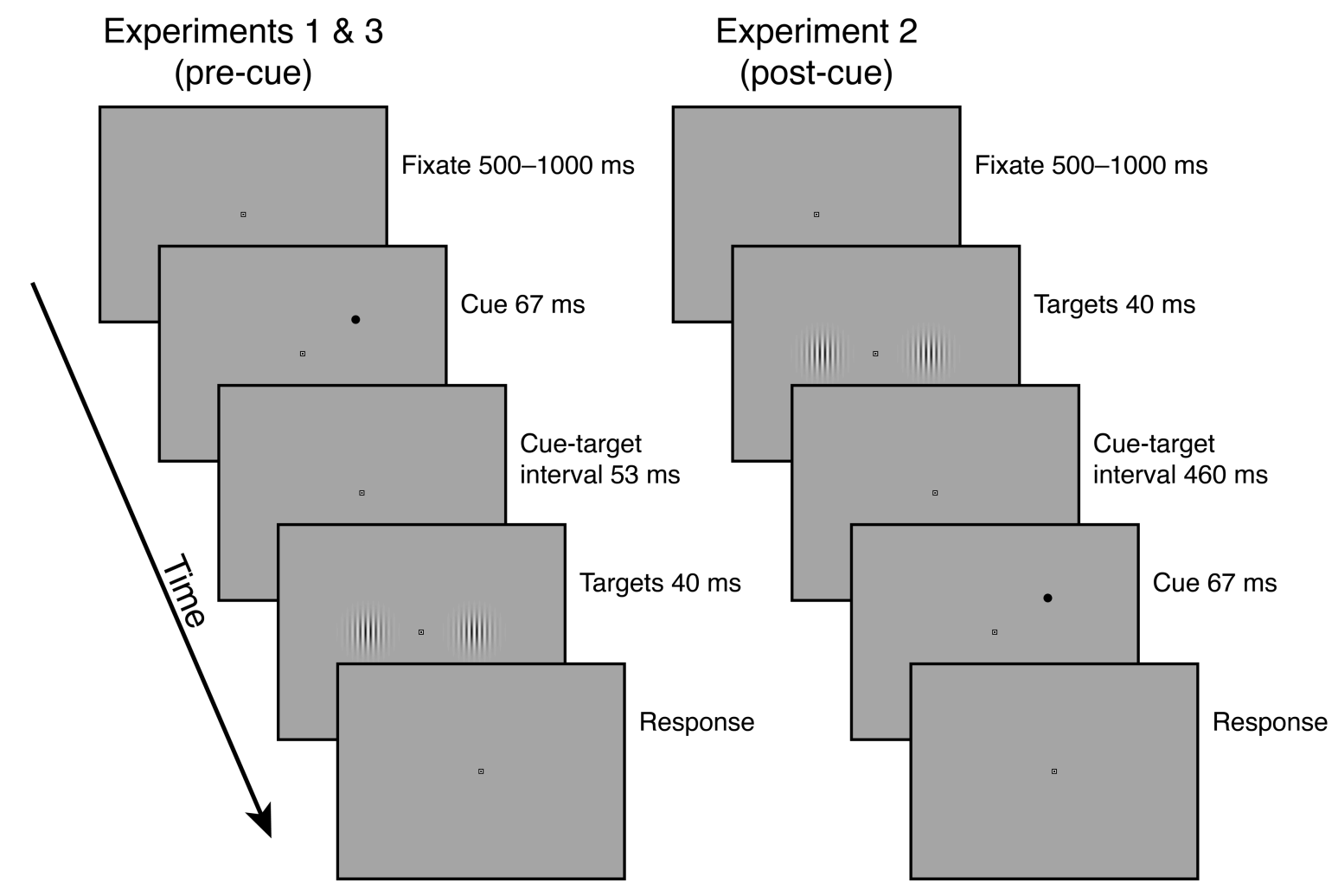


Figure 2

\section{Contrast judgment}
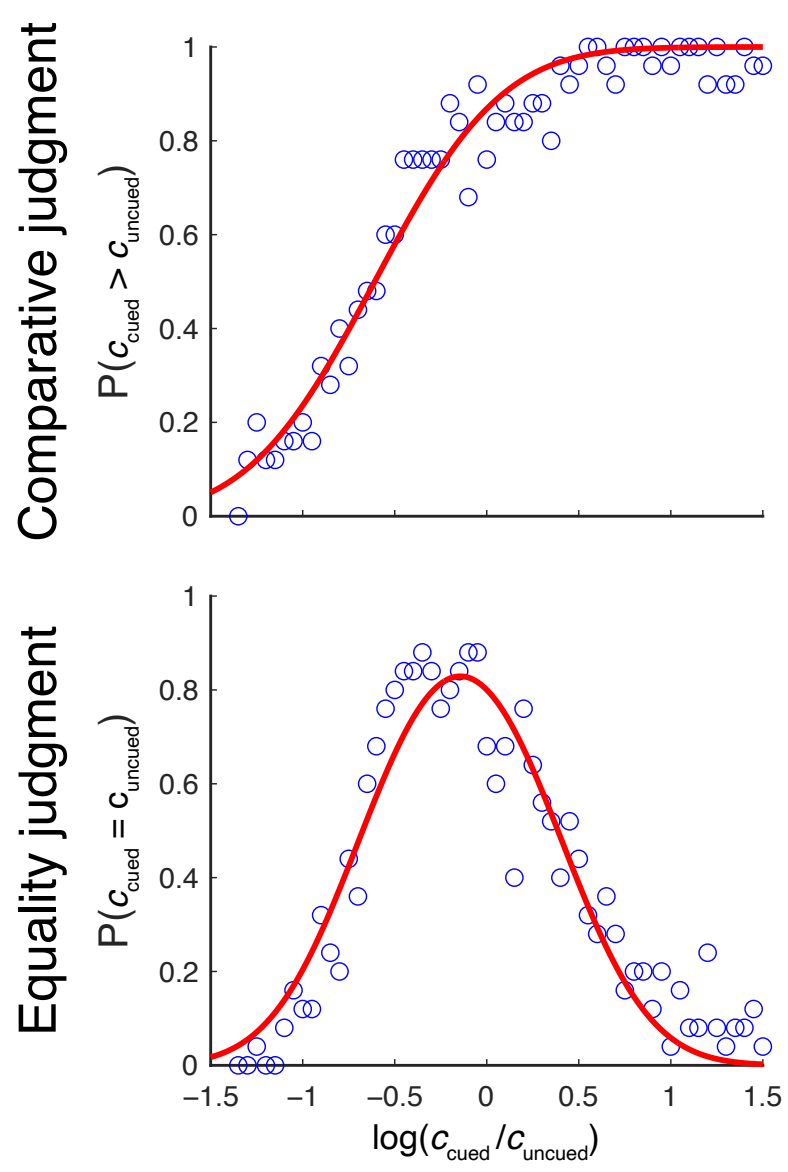

Confidence report
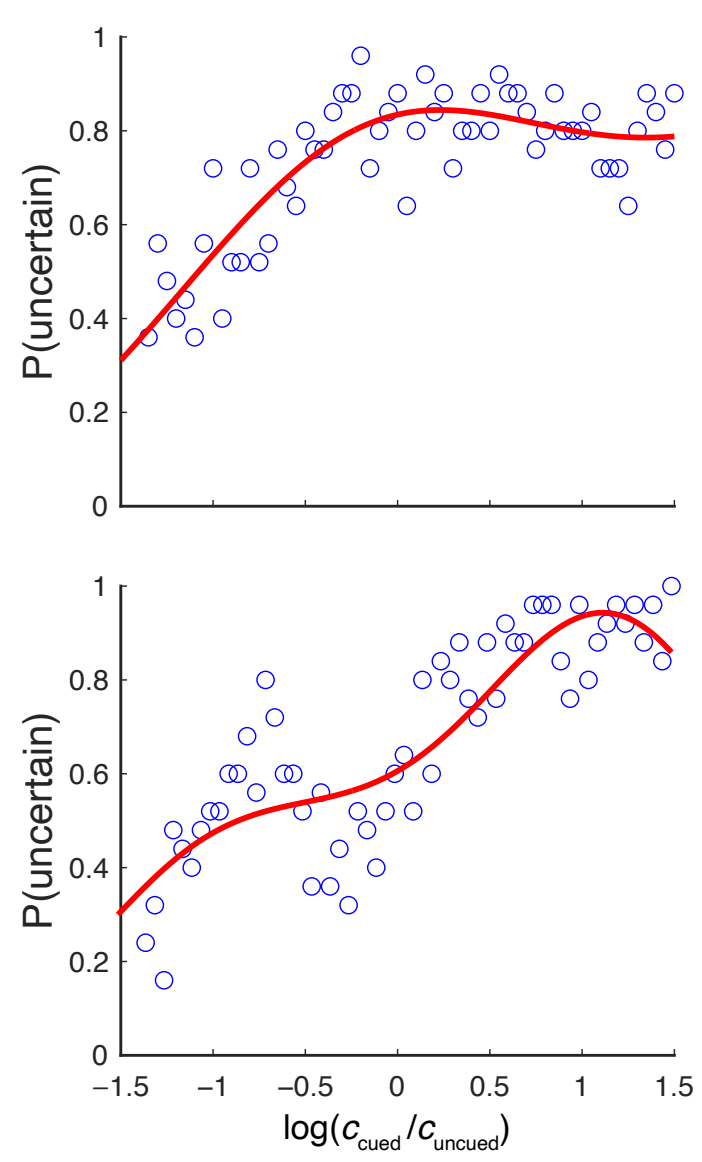
Schneider and Grigorian, Page 25

Figure 3

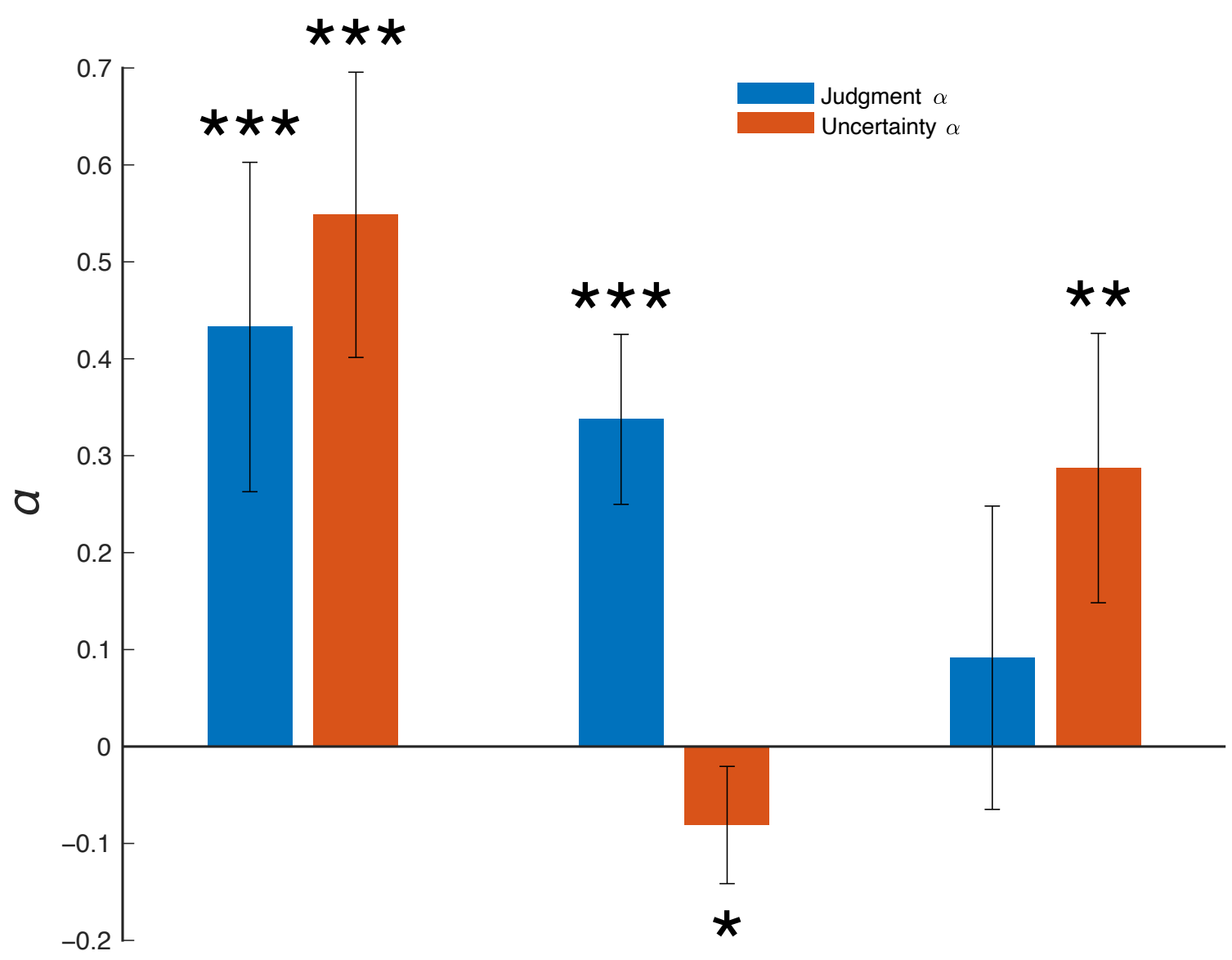

Exp. 1 pre-cue Exp. 2 post-cue Exp. 3 equality 
Schneider and Grigorian, Page 26

Figure 4
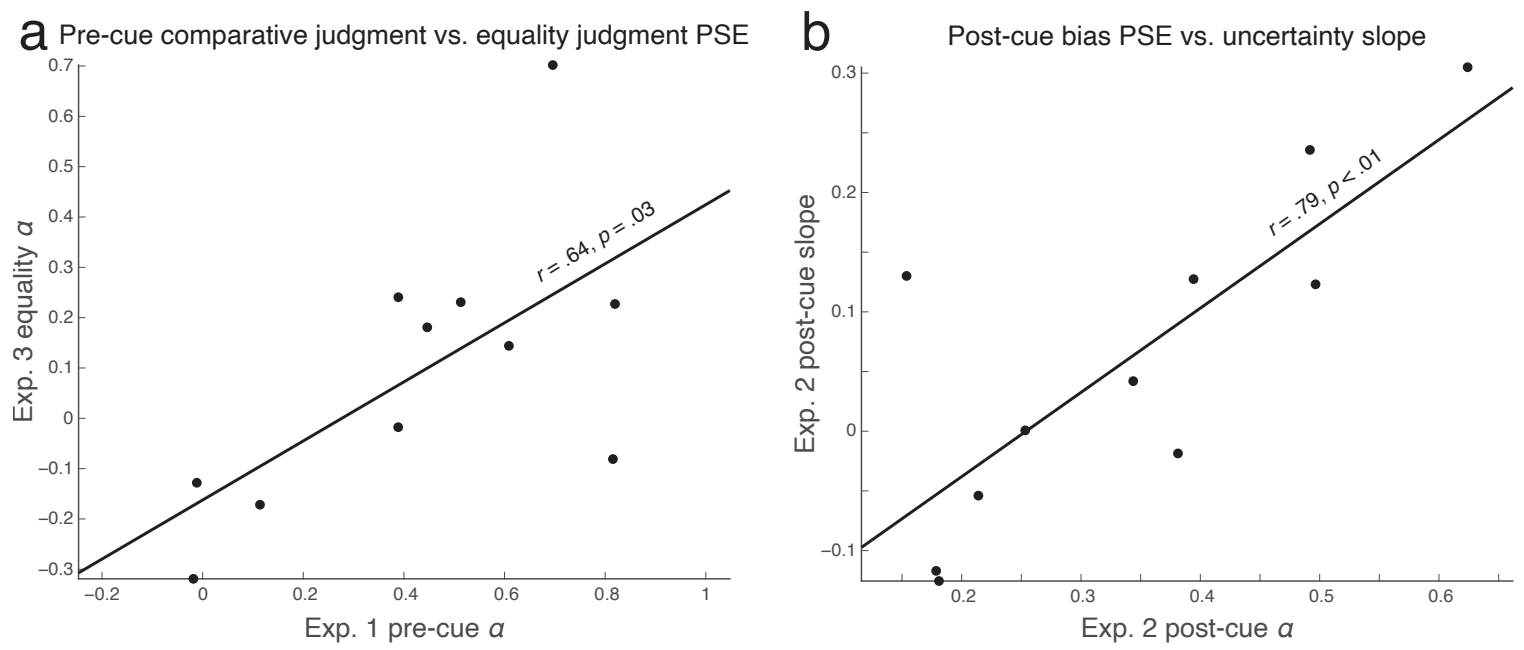
Figure 5

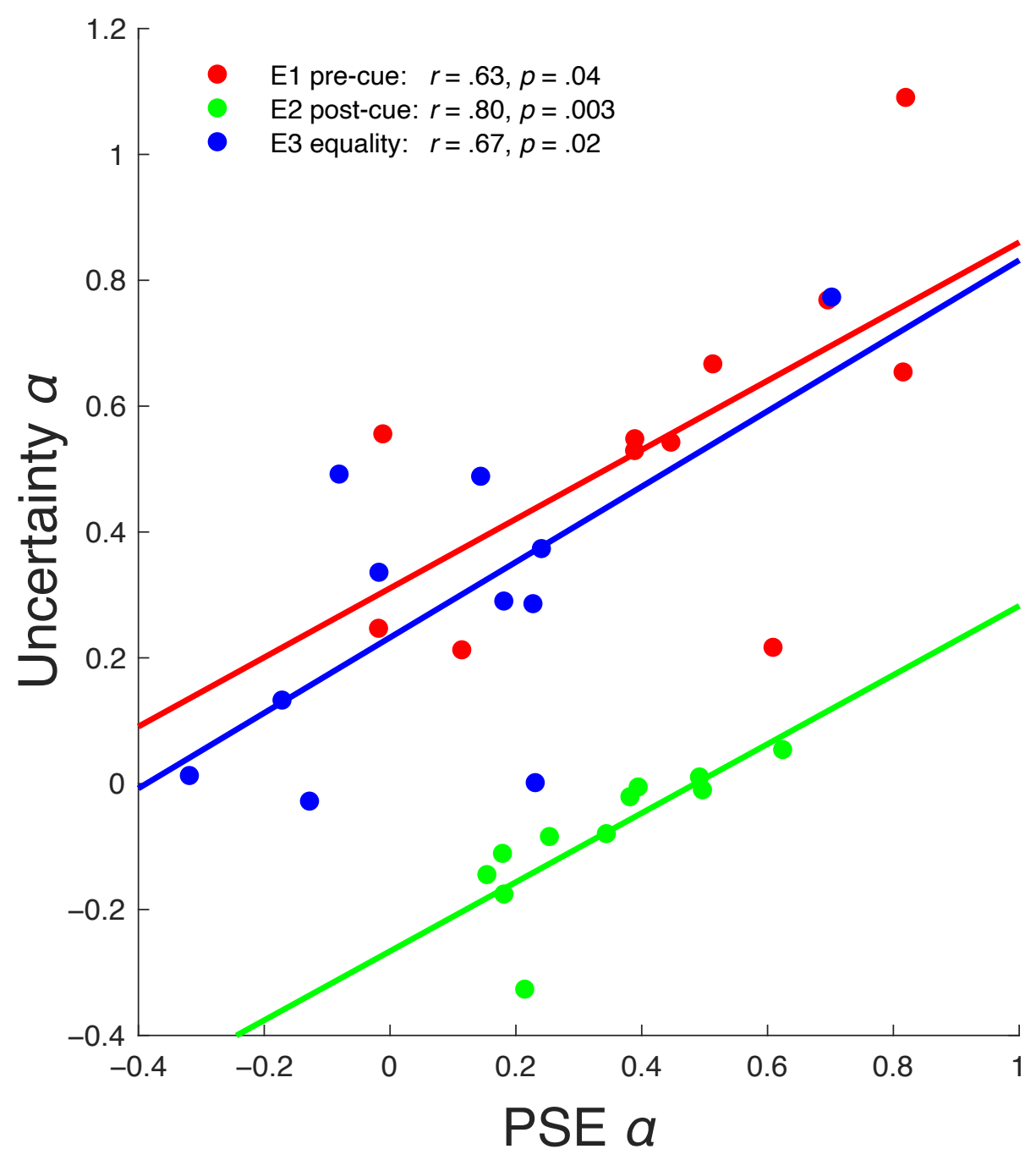


Appendix (all data from all subjects)

S1

Contrast judgment
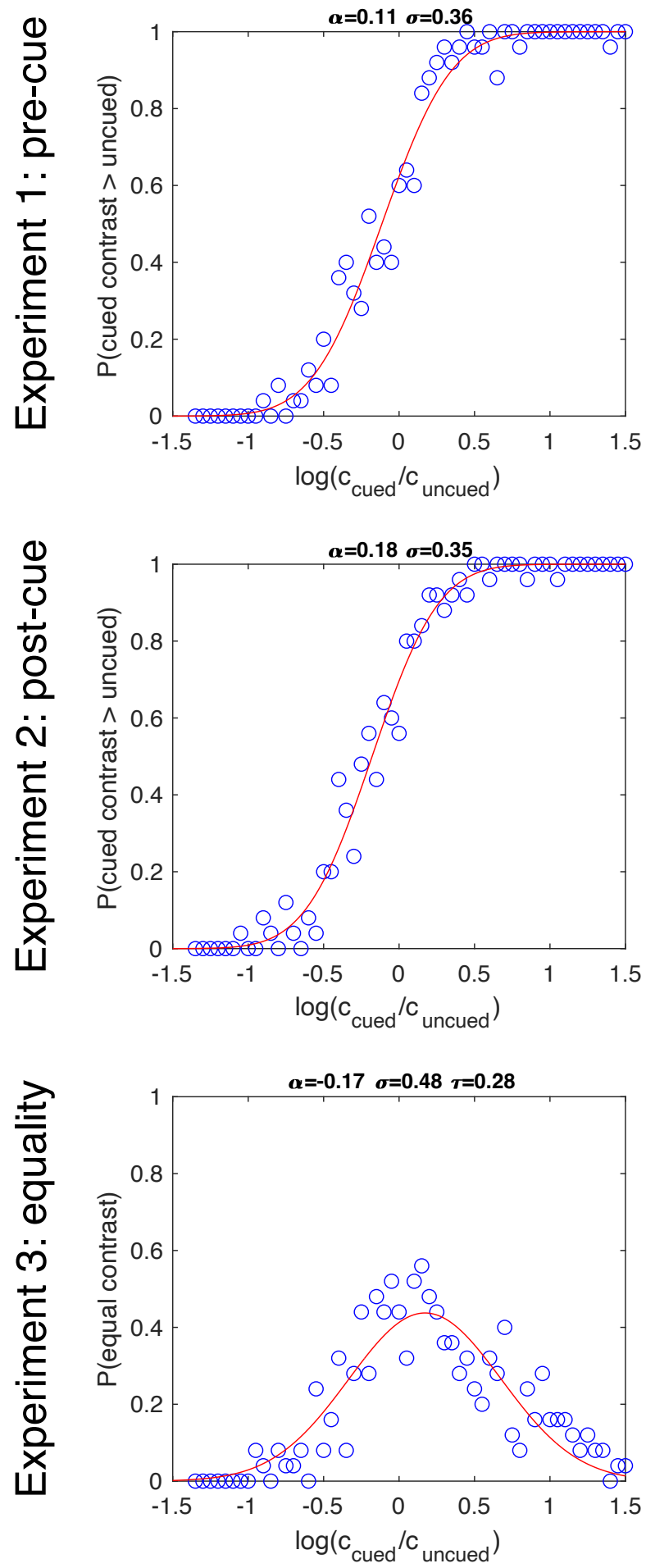

Certainty report
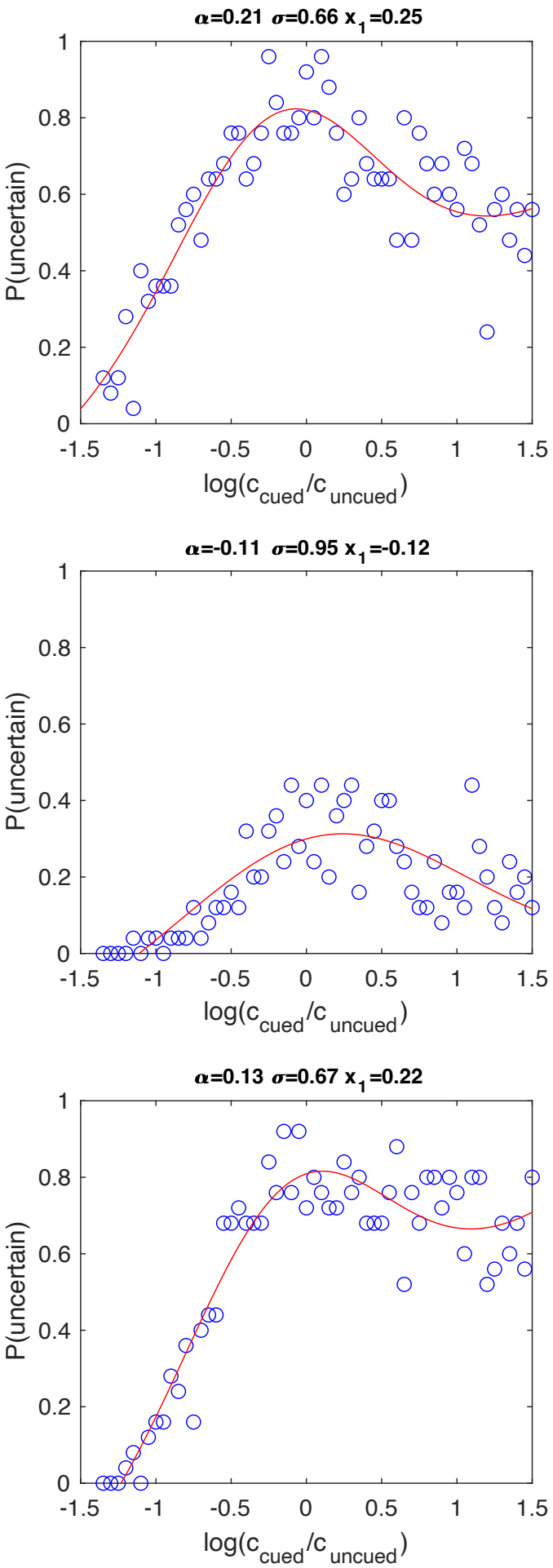
Schneider and Grigorian, Page 29

S2

Contrast judgment
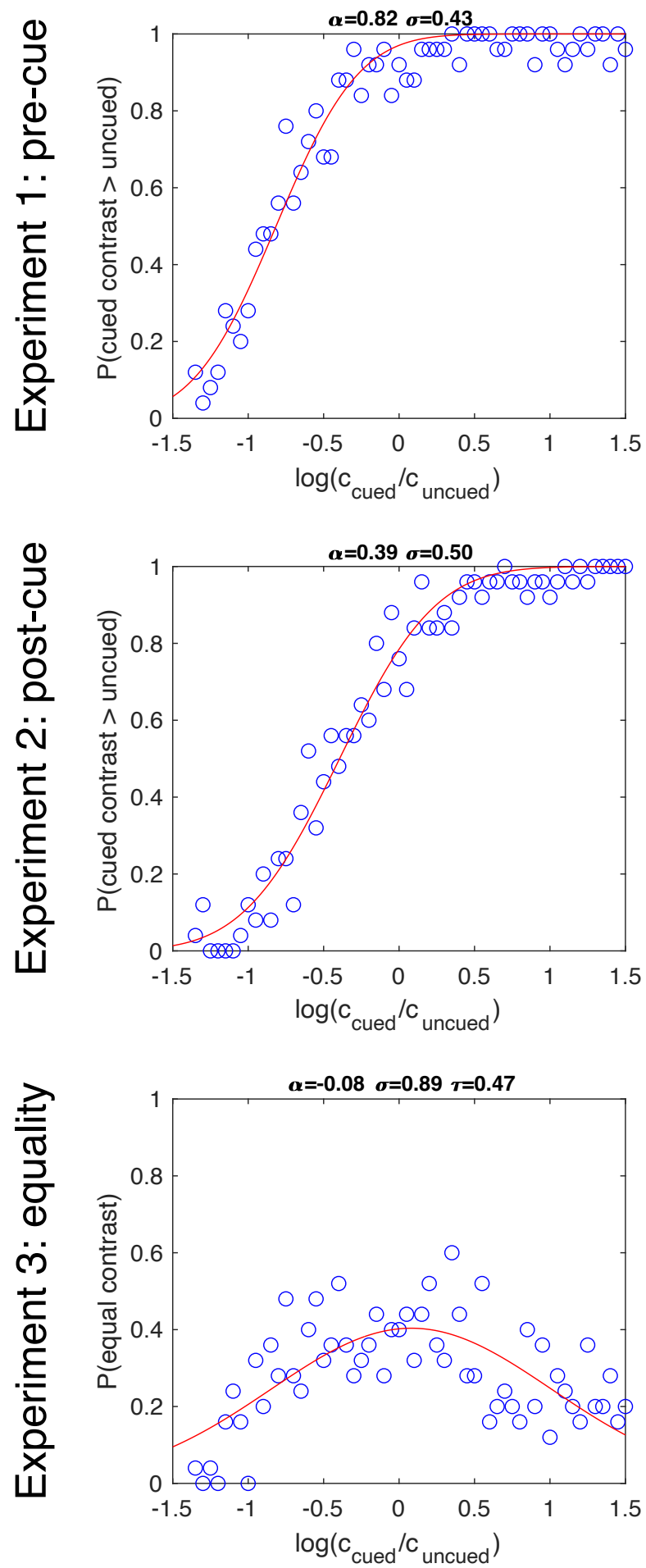

Certainty report
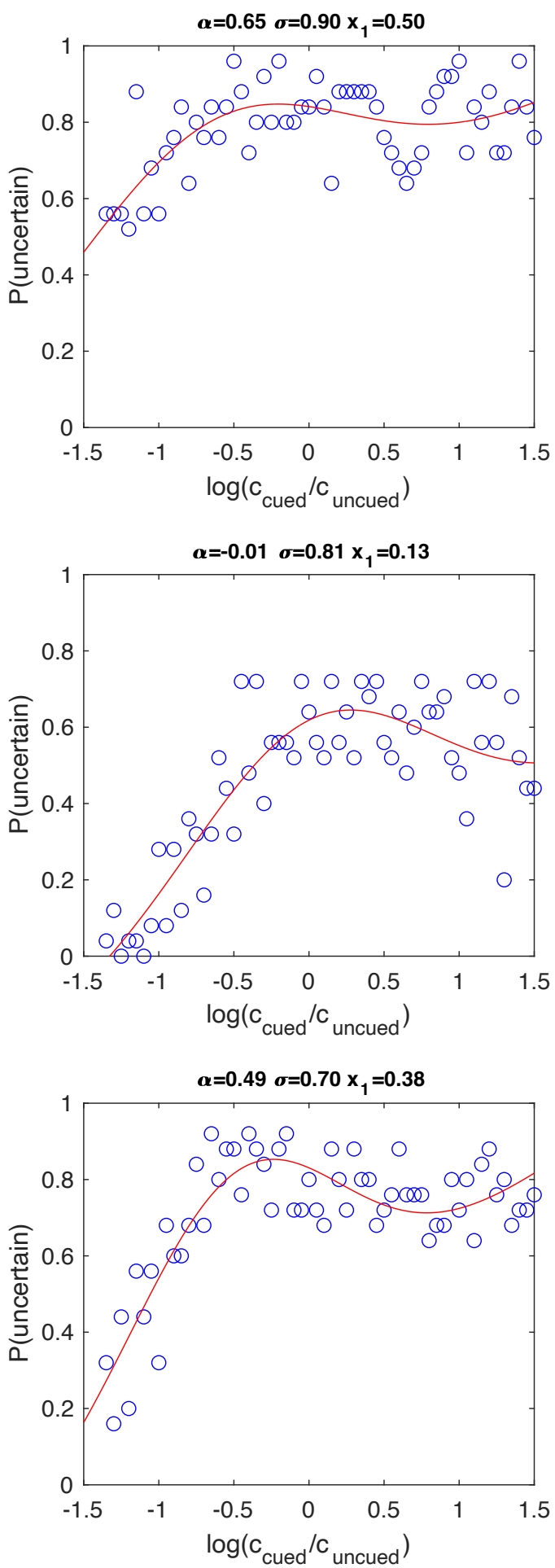
Schneider and Grigorian, Page 30

S3

Contrast judgment
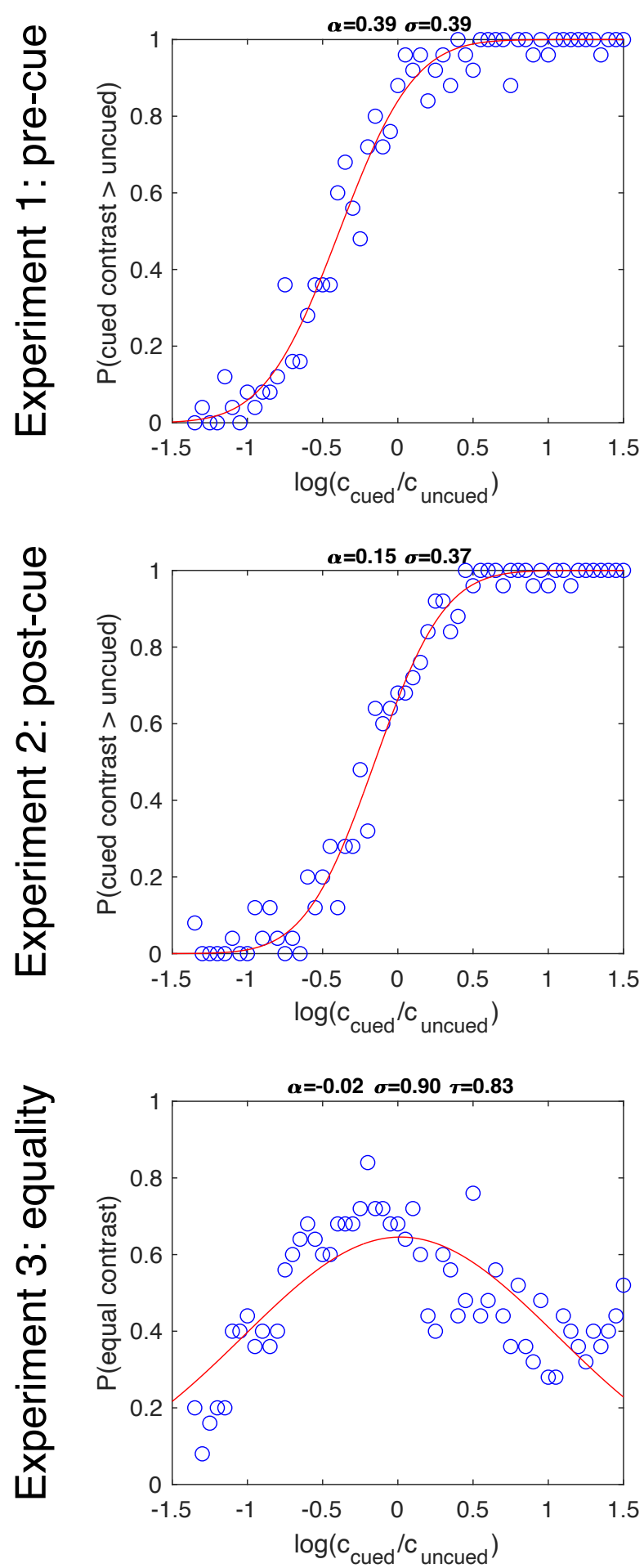

Certainty report
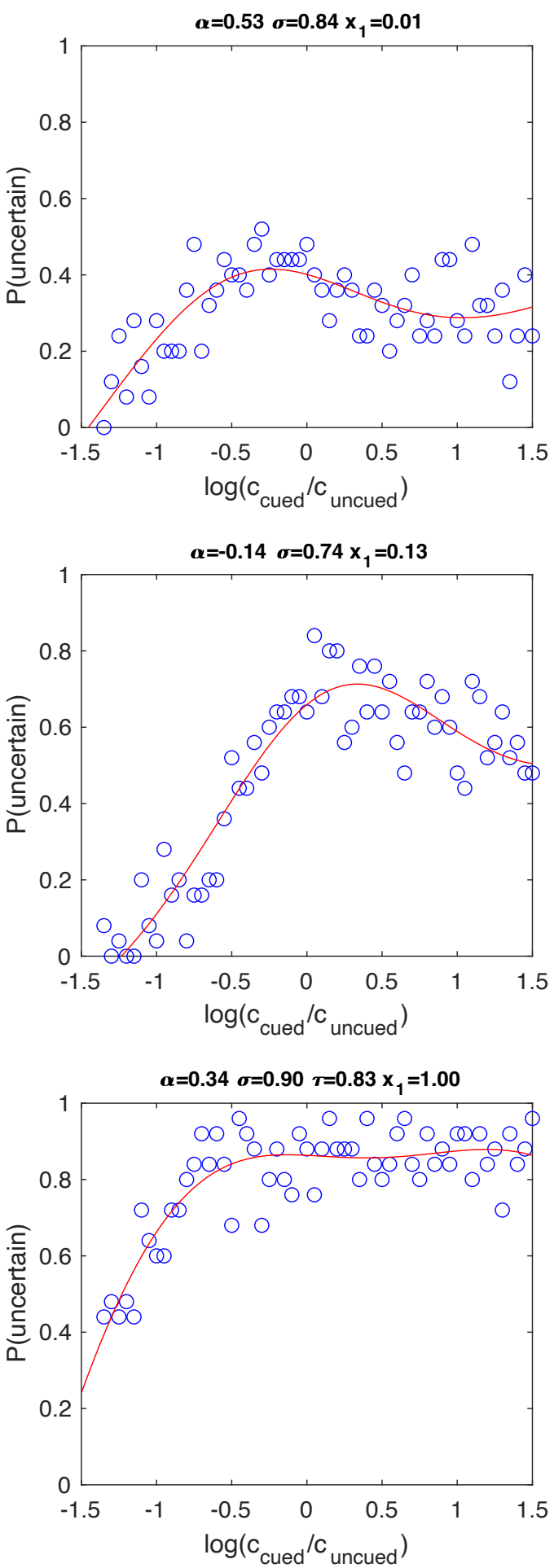
S4

Contrast judgment
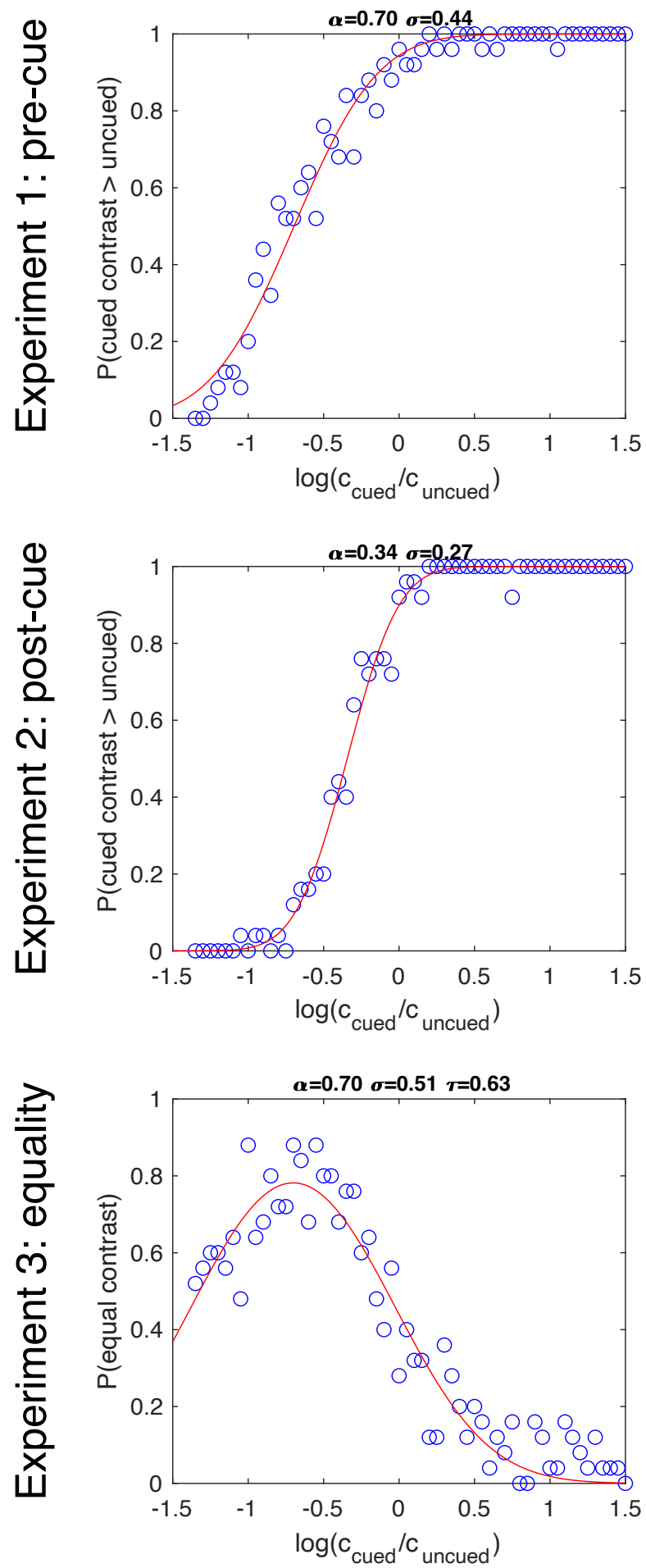

Certainty report
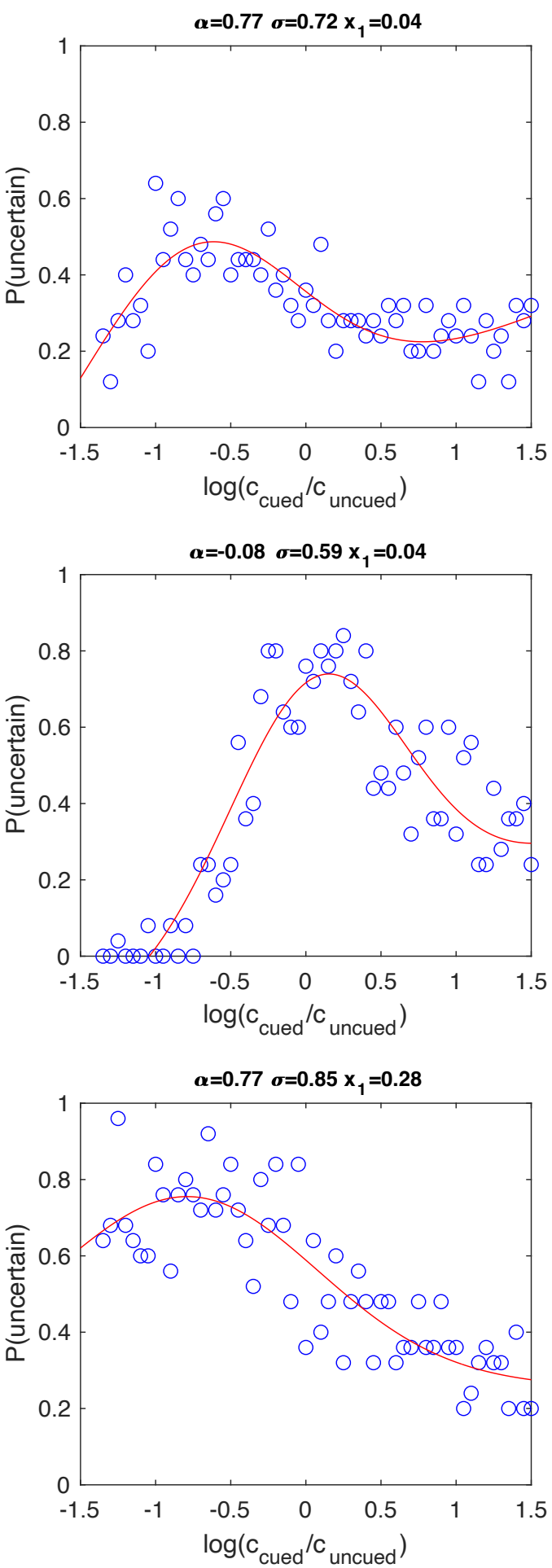
S5

Contrast judgment
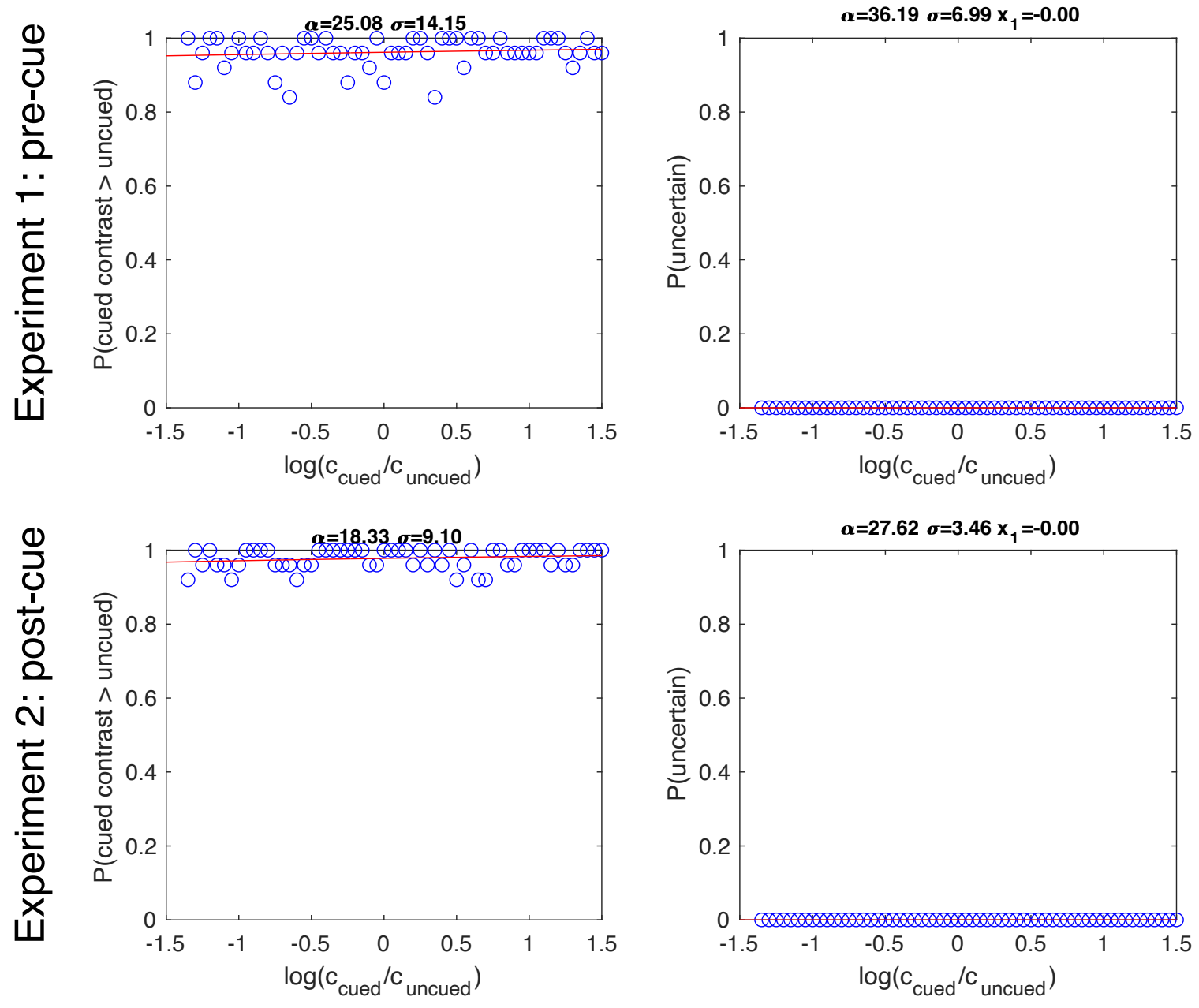

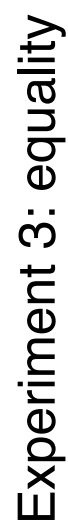

No data, subject terminated 
Schneider and Grigorian, Page 33

S6

Contrast judgment
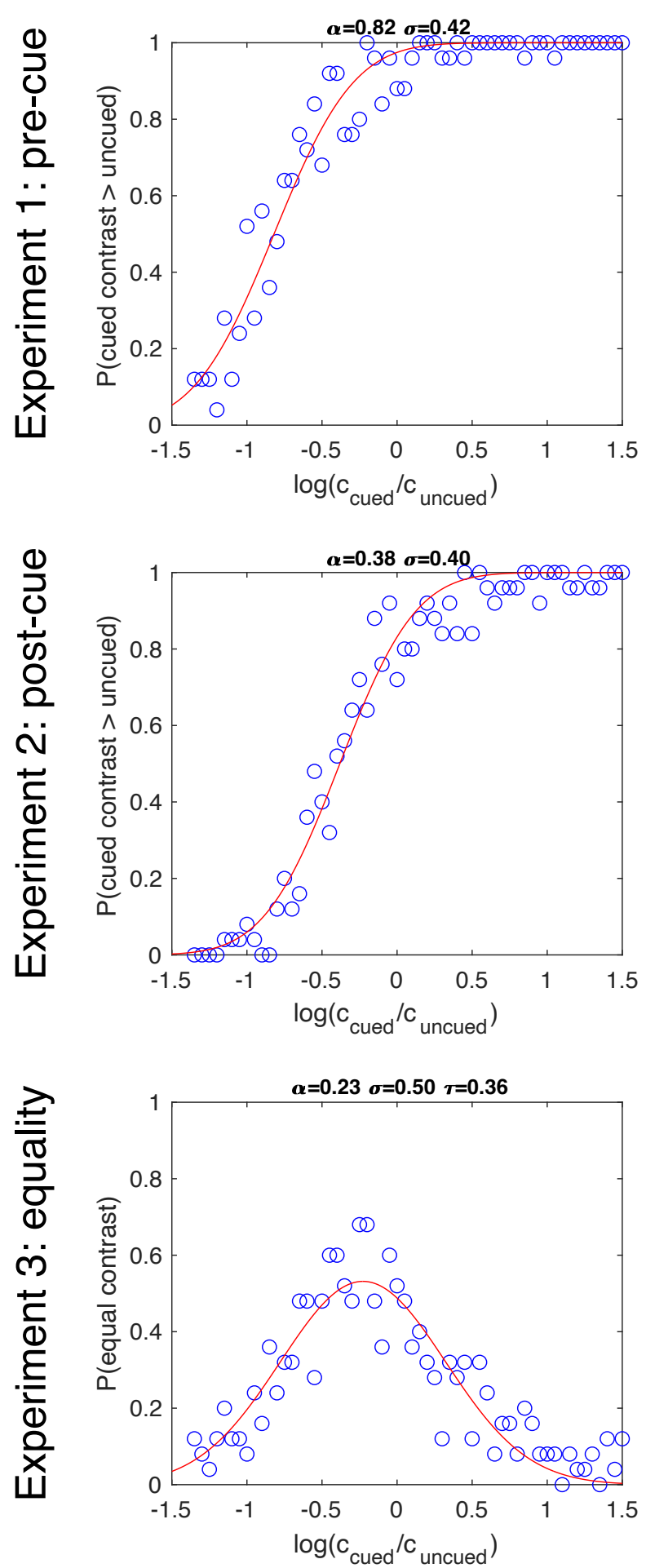

Certainty report
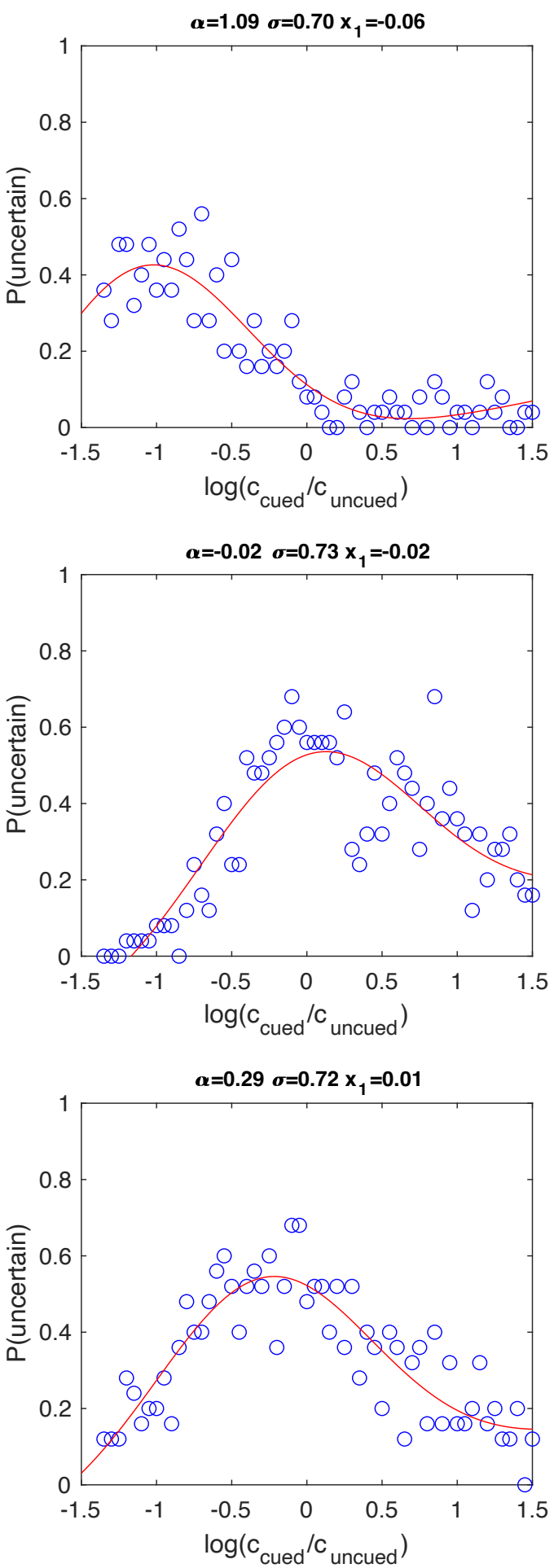
Schneider and Grigorian, Page 34

S7

Contrast judgment
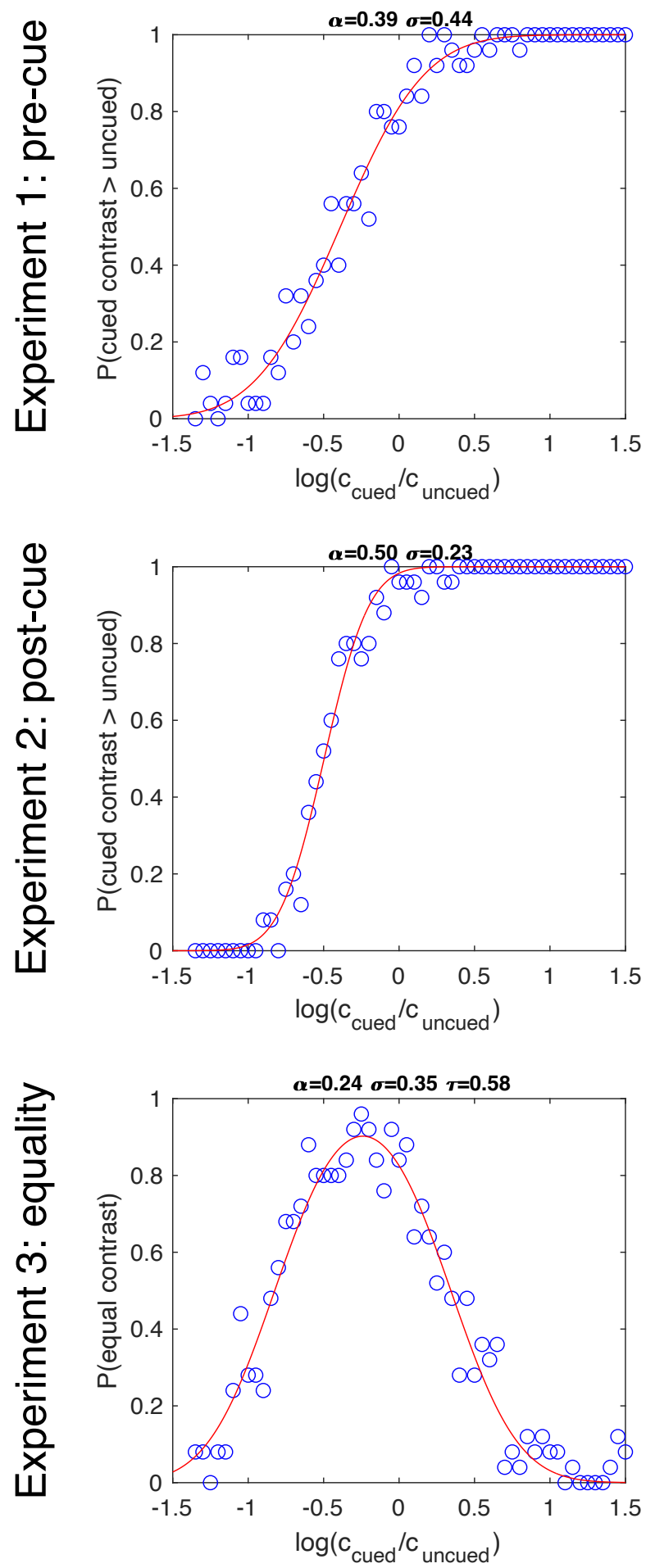

Certainty report
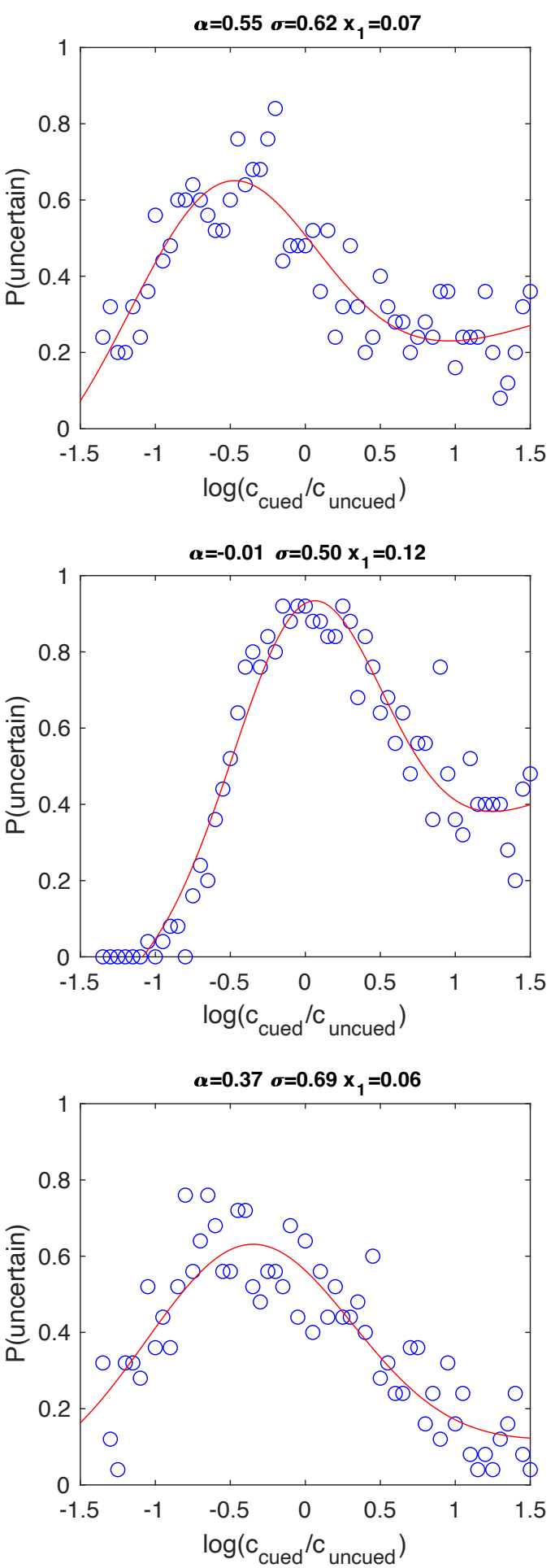
Schneider and Grigorian, Page 35

S8

Contrast judgment
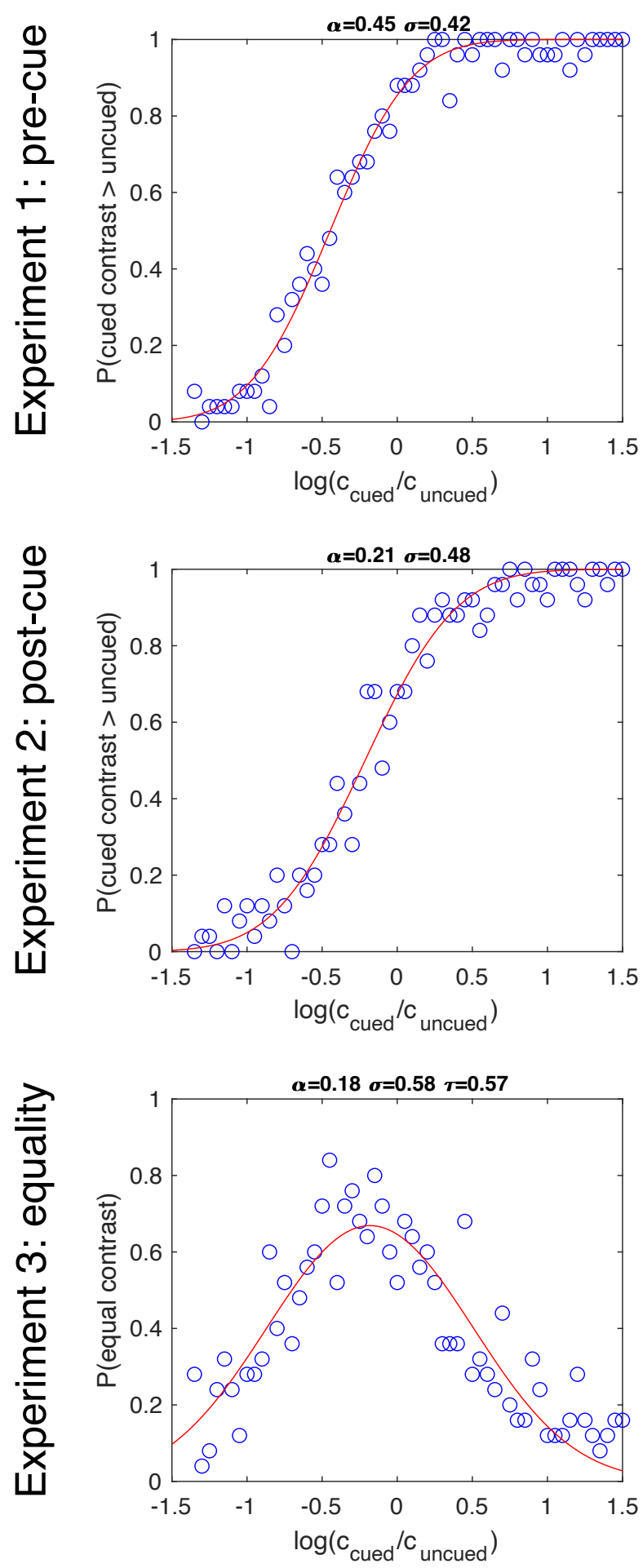

Certainty report
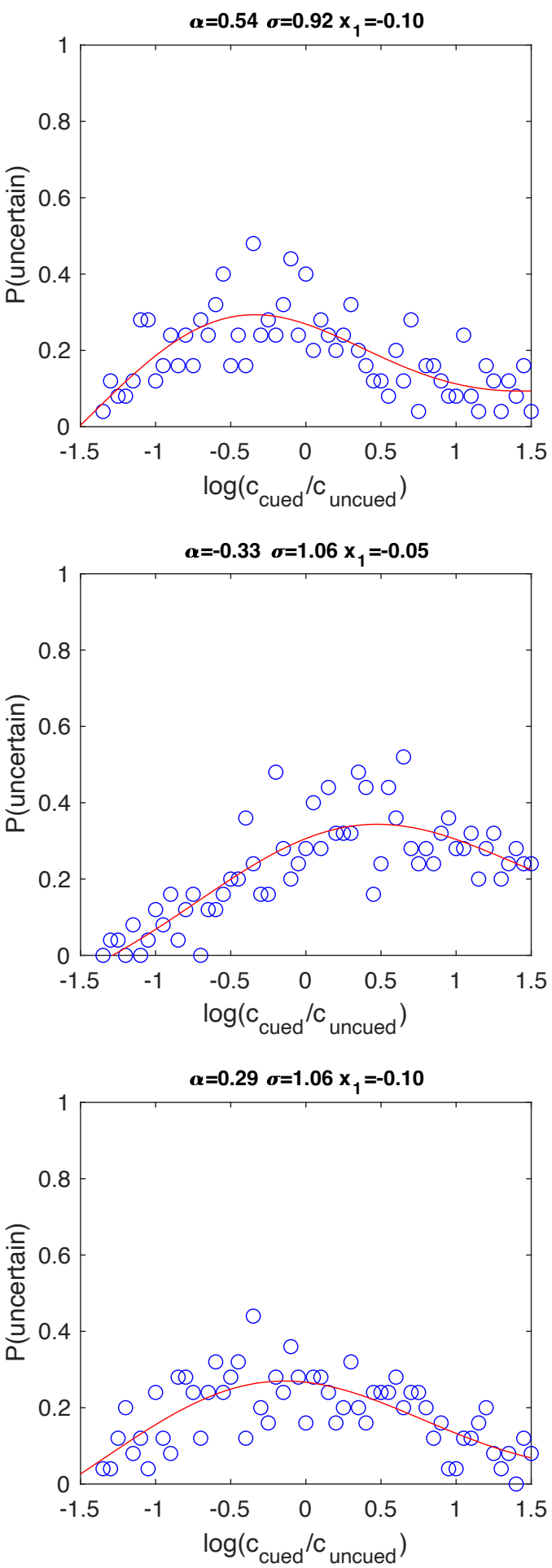
s9

Contrast judgment
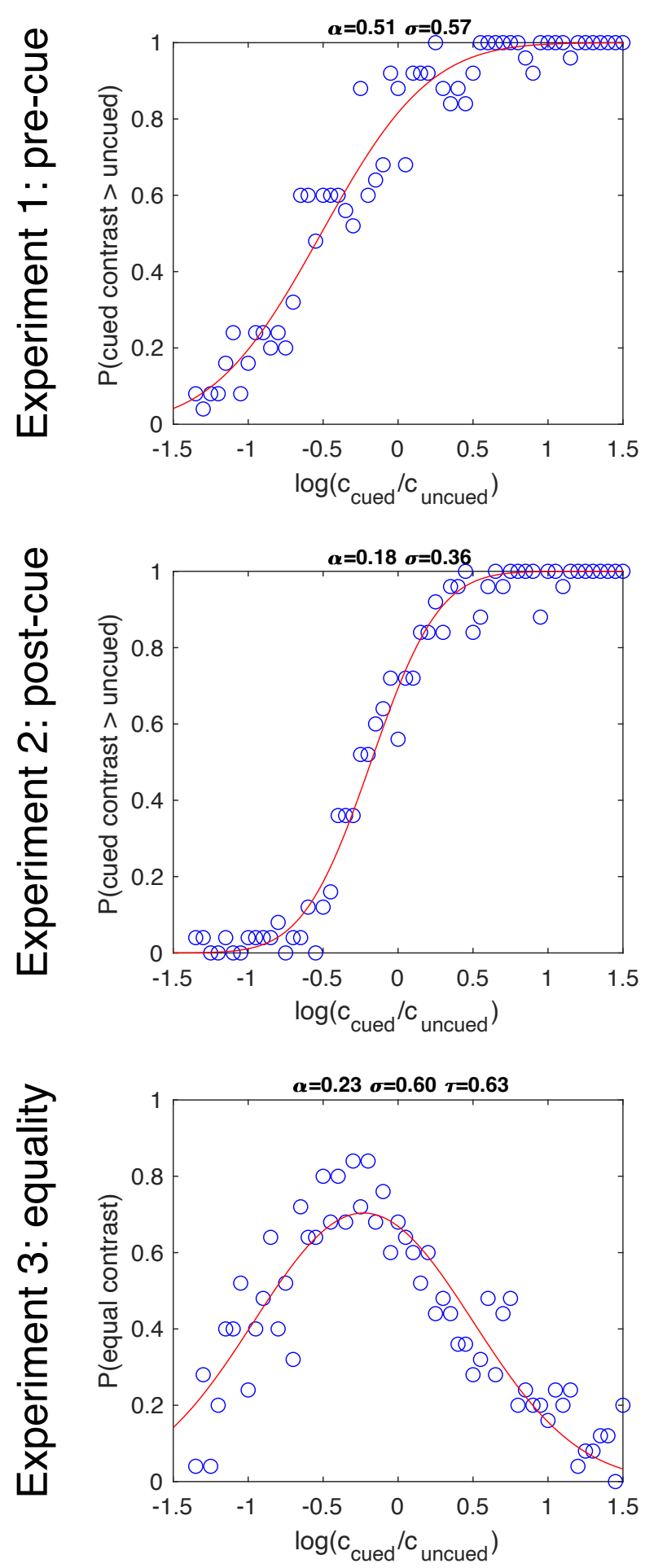

Certainty report
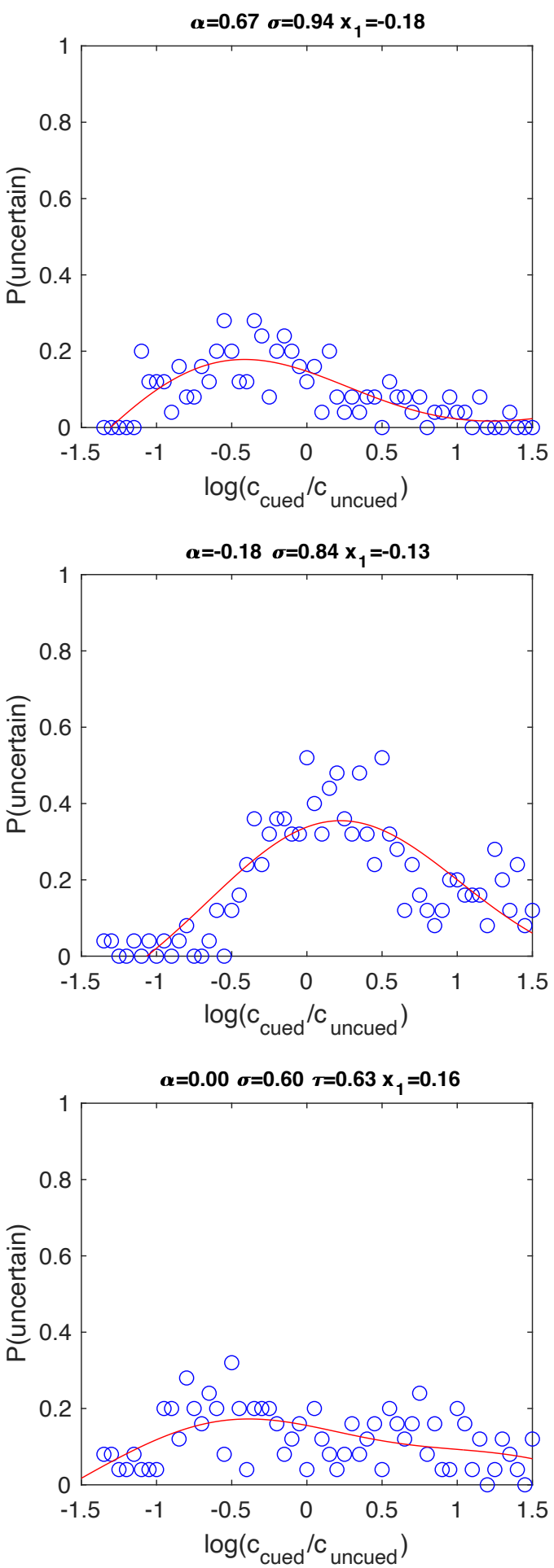
Schneider and Grigorian, Page 37

s10 Contrast judgment
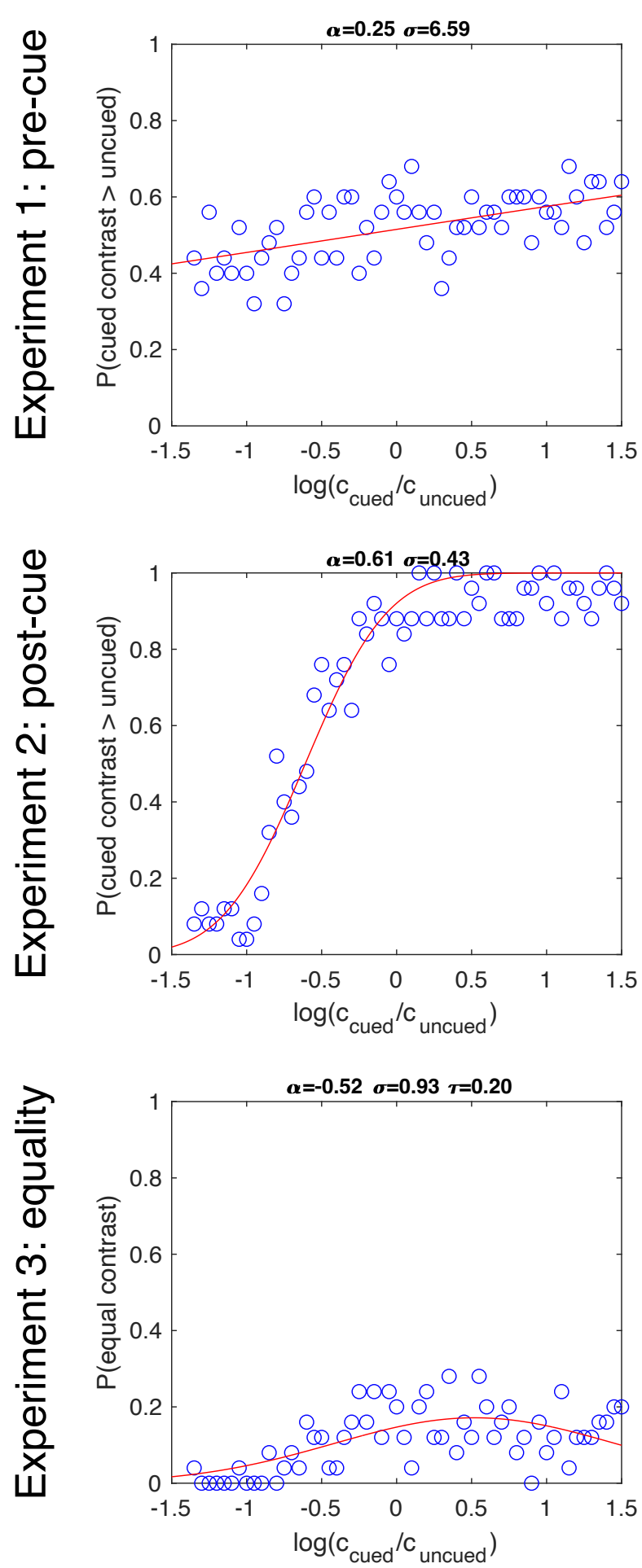

Certainty report
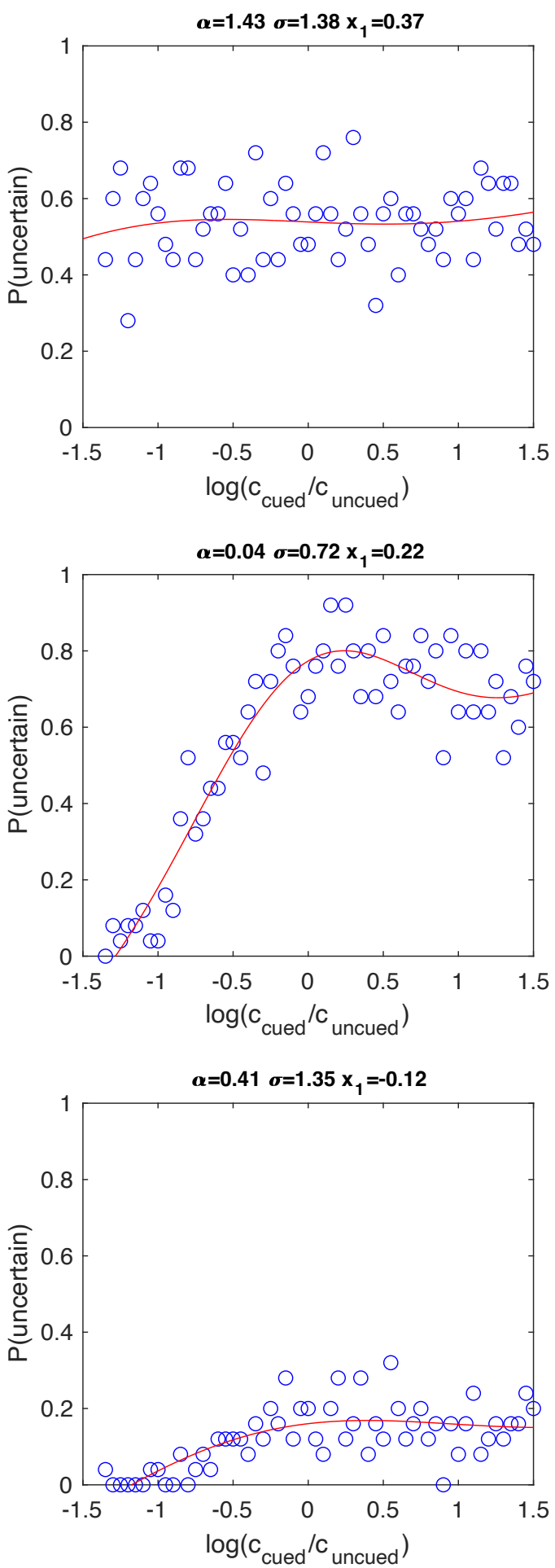
Contrast judgment
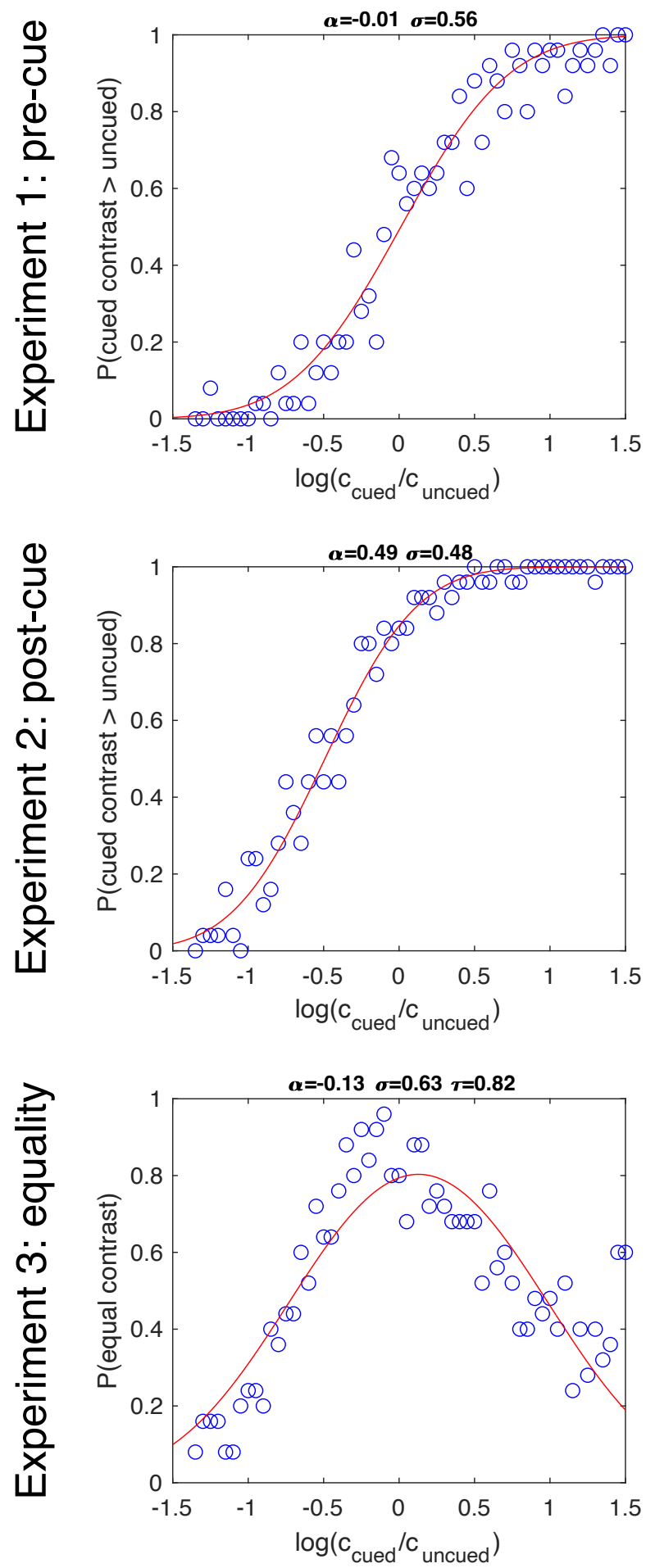

Certainty report
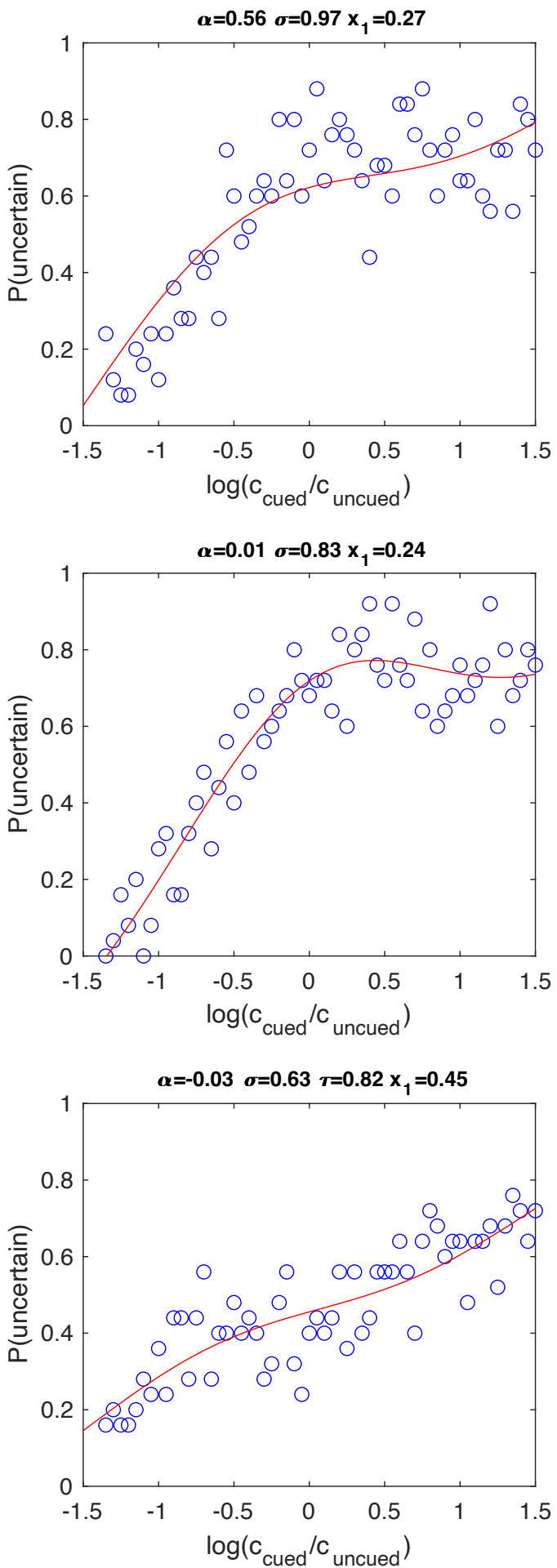
Schneider and Grigorian, Page 39

S12

Contrast judgment
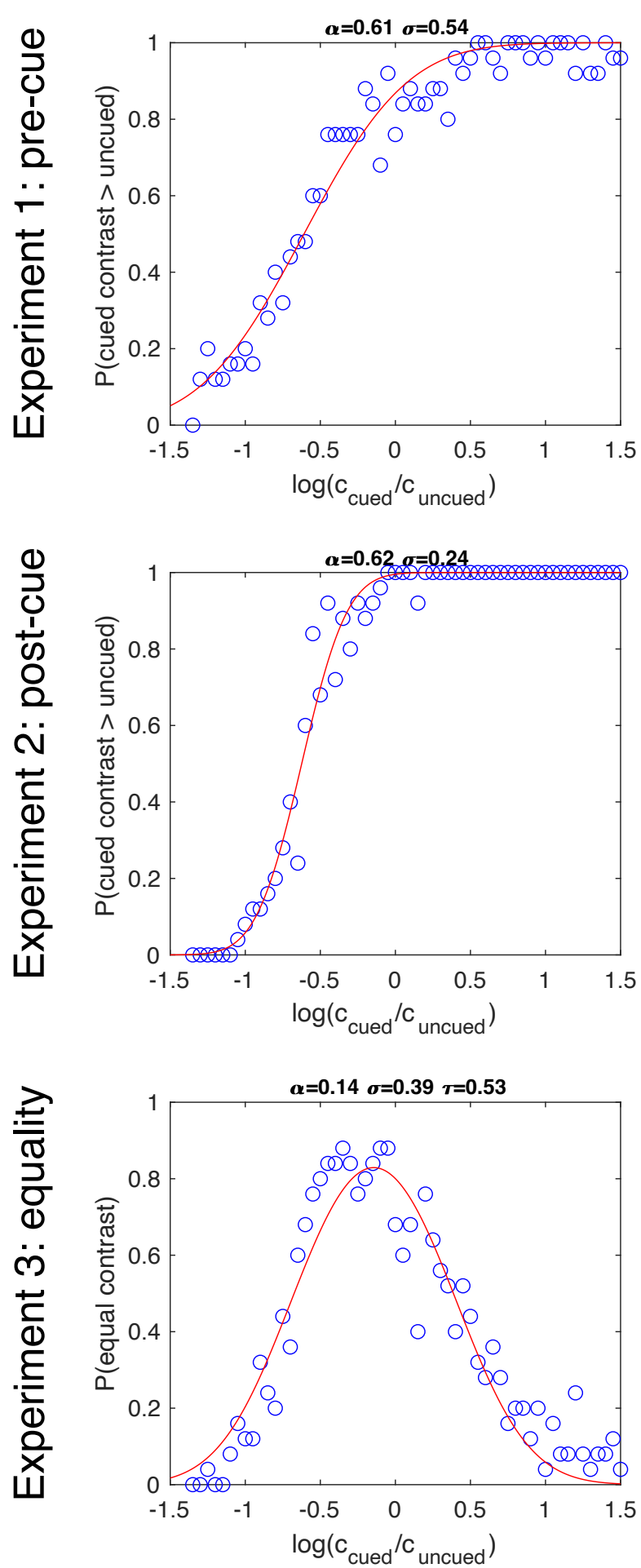

Certainty report
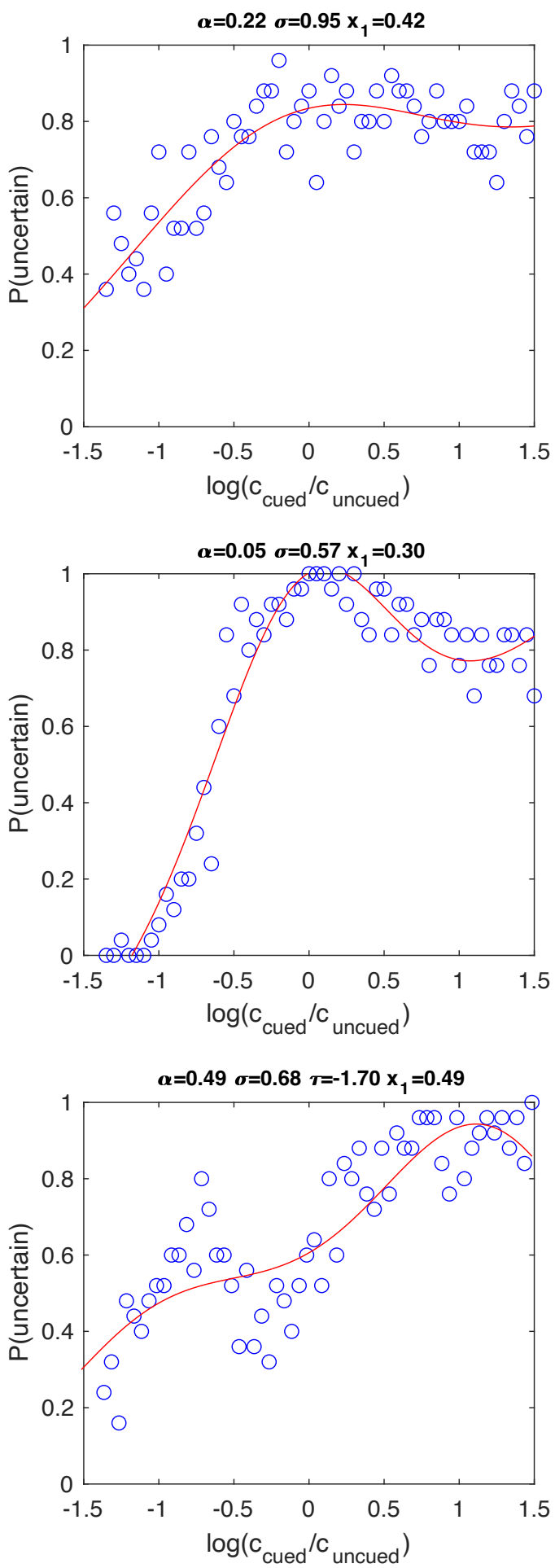
Schneider and Grigorian, Page 40

s13 Contrast judgment
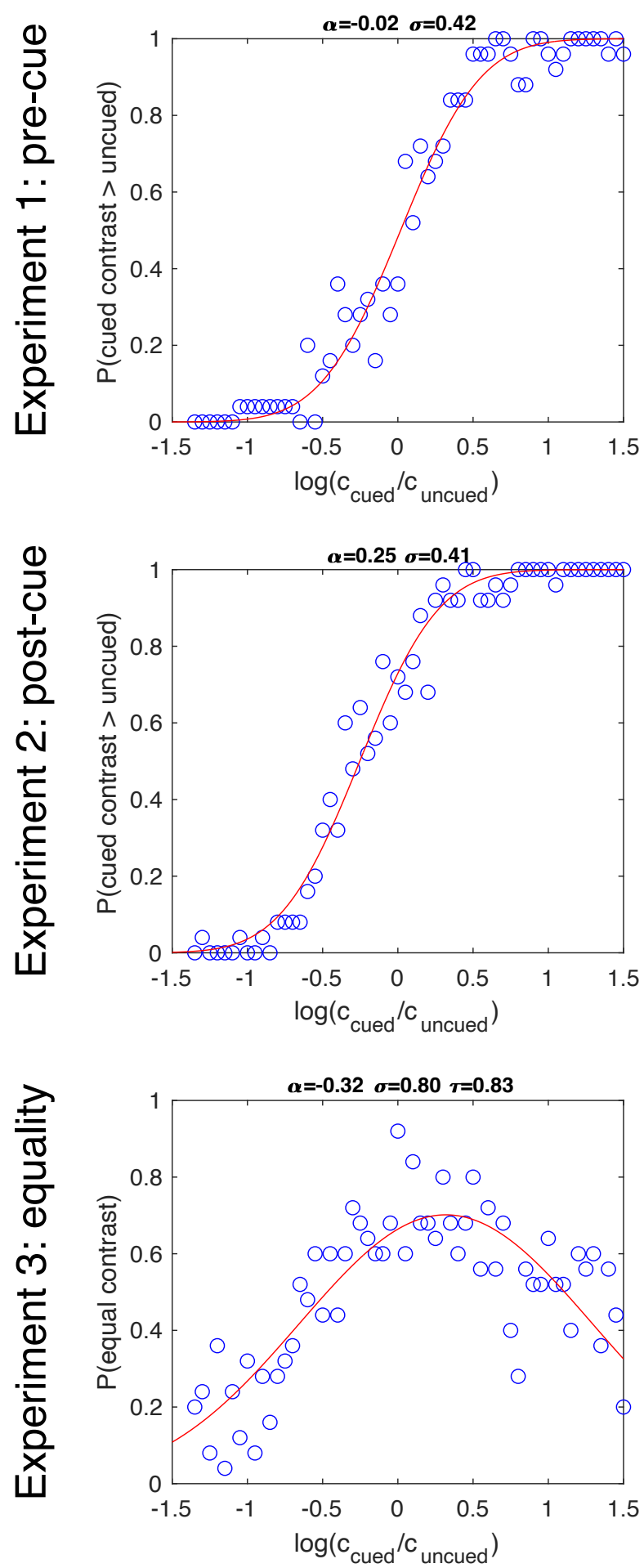

Certainty report
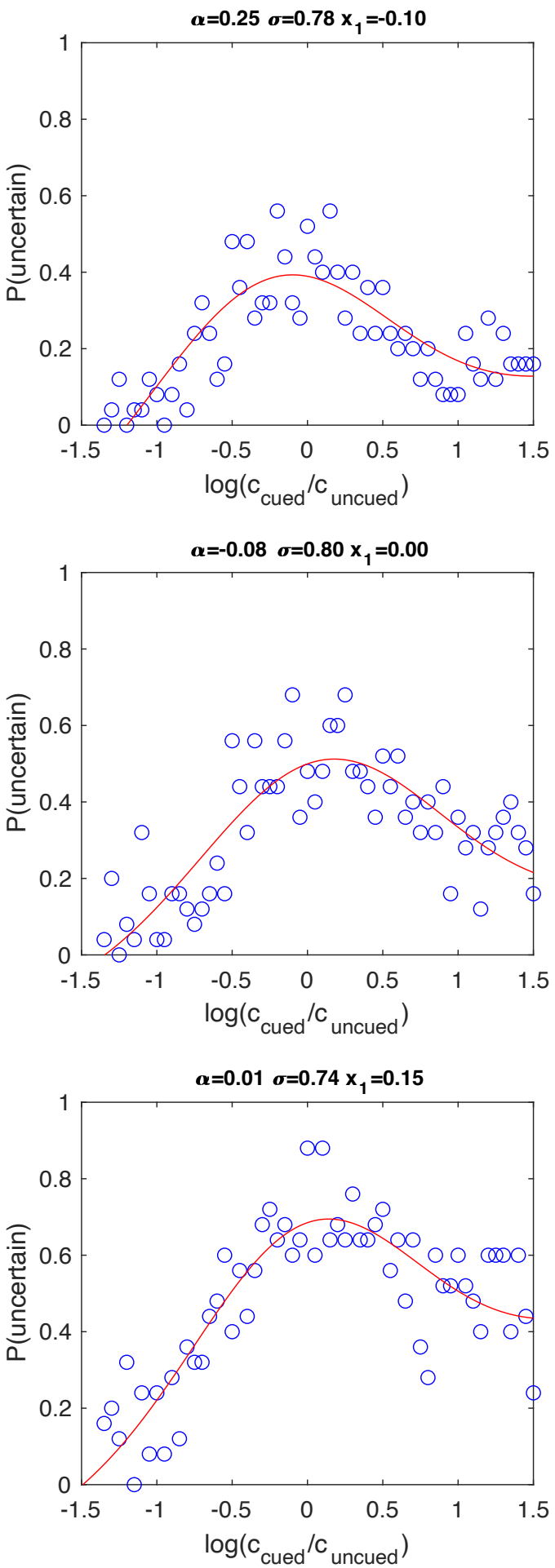
s14 Contrast judgment
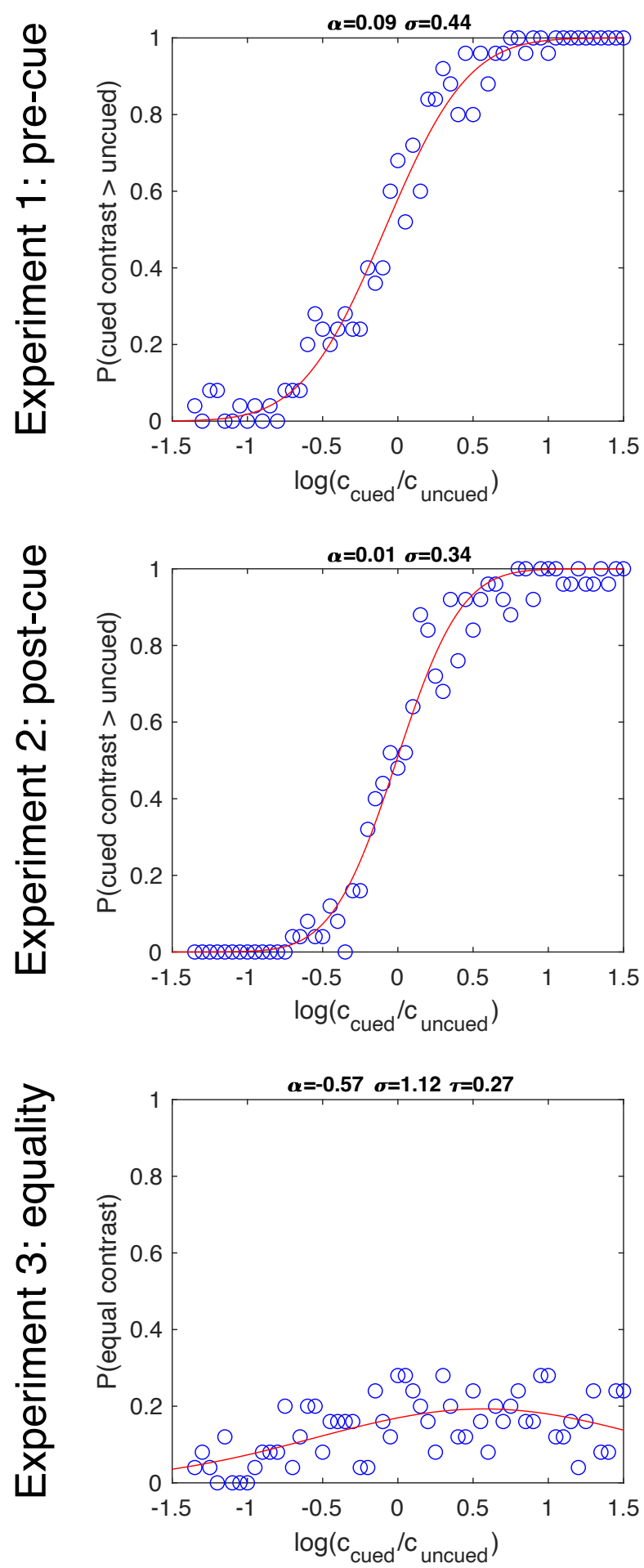

Certainty report
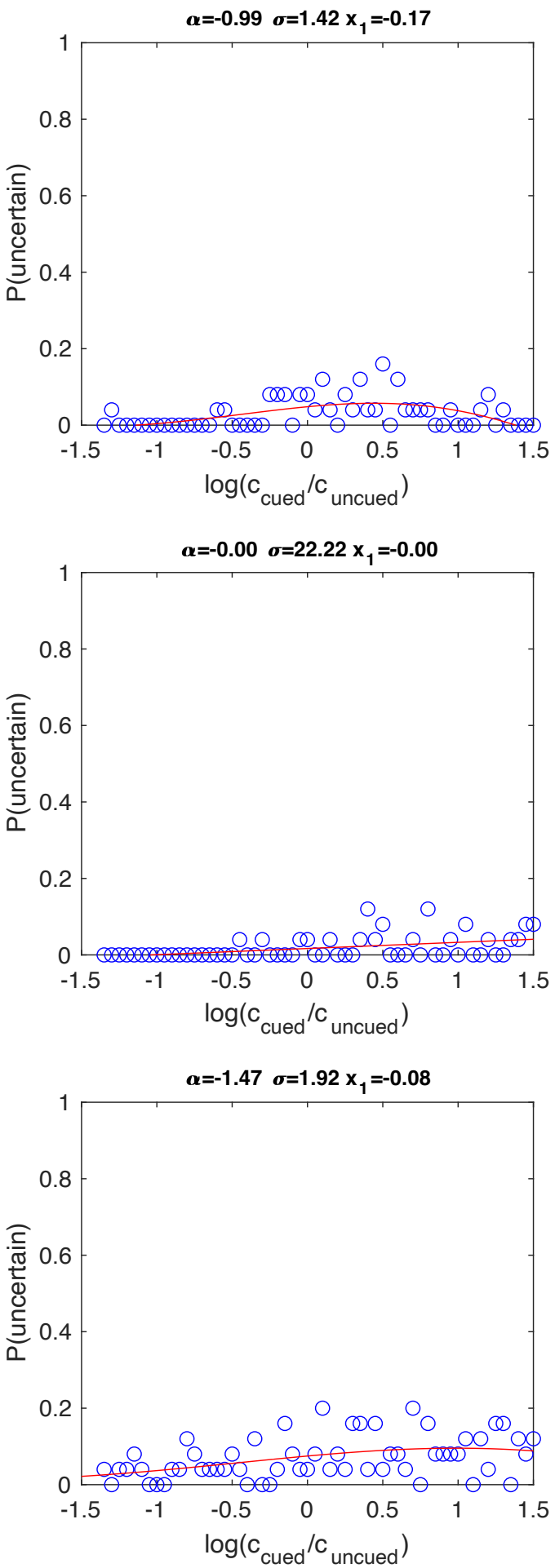